\title{
On the Initial Condition for Instanton Solutions
}

\author{
P. Štovíček \\ Laboratory of Theoretical Physics, Joint Institute for Nuclear Research, \\ SU-141980 Dubna, USSR*
}

Received January 24, 1989; in revised form March 2, 1990

\begin{abstract}
To each gauge equivalence class of both local and global framed (in the sense of Donaldson) self-dual solutions with the gauge group $\mathbf{U}(\mathbf{r})$ there is related the unique canonical initial condition (in the sense of Takasaki) and in this way the gauge freedom is eliminated. A geometric interpretation is given and consequently the complete transcription of the ADHM construction into the inverse scattering formalism is derived. As an application, an injection holomorphic mapping of the instanton moduli space into a finite-dimensional complex vector space is described and the loop group action on the transition functions is discussed. The results suggest the possibility of a new description of the framed instanton moduli spaces directly as algebraic sets.
\end{abstract}

\section{Introduction}

It is well known that the self-dual Yang-Mills equations admit two different approaches: one initiated by Ward [1] and based on the Penrose twistor transformation and the other, going back to Yang [2], Belavin and Zakharov [3], based on the inverse scattering method. The former one, insisting on global methods (cf. [4]), has succeeded in the ADHM construction [5]. The inverse scattering approach is essentially local. It insists on expressions in local coordinates and consequently it enables one to apply some more general methods such as the Bäcklund transformation $[6,7]$, the construction of an infinite algebra of symmetries due to Dolan [8] and Chau, Ge, Sinha and Wu [9] and the solution of the initial value problem due to Takasaki [10].

This paper addresses the initial condition in the sense of Takasaki and its geometric interpretation in the global case. The starting point is a more detailed discussion of the gauge transformations in the local formulation. The main point is to eliminate completely the gauge freedom. The geometric interpretation suggests a new way to describe the framed instanton moduli spaces (in the sense of Donaldson [11]) directly as algebraic sets. We recall that in the ADHM

* On leave from: Department of Mathematics, Faculty of Nuclear Science, Czech Technical University, Prague, Czechoslovakia 
construction a finite-dimensional Lie group still remains as a reminiscence of the infinite-dimensional group of gauge transformations. However, this problem here is only suggested and illustrated by an example but not completely solved.

More precisely, this paper aims to present the following results:

(1) In the inverse scattering formalism, there is stated explicitly a condition which enables one to distinguish a special "canonical" solution in each gauge equivalence class (Theorem 3.3). Particularly, one can introduce the notion of the canonical initial condition. Since the initial value problem has the unique solution, the gauge freedom is eliminated in this way. It follows that the space of gauge equivalence classes of local solutions has an extremely simple structure; it can be identified with $\mathbb{R}^{\infty}$ (relation (3.16)). A gauge transformation resulting in the canonical solution is described explicitly (relations (3.14), (3.18)). As a corollary, one gets an embedding of the framed instanton moduli space into $\mathbb{R}^{\infty}$ (Proposition 3.4 and the remark following it).

(2) In the twistor framework, we describe a geometric construction relating the canonical initial condition to a distinguished and again called canonical transition function (Chap. 4). This means that to each gauge equivalence class of global framed instanton solutions there is related the unique matrix valued function on $\mathbb{P}^{3}$ with some special properties (Theorem 4.4; Proposition 4.3 and relations (4.3), (4.4)). Almost all deliberations associated with the construction remain valid even in the more general case of framed holomorphic vector bundles on $\mathbb{P}^{n}, n \geqq 2$.

(3) Using this construction, one is able to obtain explicit expressions for the canonical initial condition of the ADHM instanton solutions [relations (5.10)] and consequently to give the complete transcription of the ADHM construction into the inverse scattering formalism (Theorem 5.3). To the author's knowledge, despite the fact that the ADHM construction has become classical, such a transcription was nowhere derived and published yet. Hopefully, it will enable one to check and further develop some previous concepts such as the Bäcklund transformation.

(4) In Chap. 6, we make use of the one-to-one correspondence between the moduli space of framed instanton solutions on $\mathbf{S}^{4}$ and the moduli space of framed holomorphic bundles on $\mathbb{P}^{2}(\mathbb{C})$ which has been proved by Donaldson in [11]. The latter space is a complex manifold and we describe two ways of its holomorphic embedding into a finite-dimensional complex vector space (Propositions 6.2, 6.3). The latter embedding opens, in principle, the way to a new description of the framed instanton moduli spaces. We are able to give some more concrete results for the $\mathbf{U}(2)$ - gauge group (Proposition 6.4).

(5) Following Crane (cf. [12]), we consider in Chap. 7 the loop group action on the instanton transition functions. Applying the results of Chap. 3, we discuss the infinitesimal action on the one-instantons. The loop group action is gauge dependent and well defined on the local transition functions provided the reality condition (7.8) is satisfied. But the infinitesimal action is tangent to the embedded one-instanton moduli space only under some special conditions and, if it is the case, it coincides with an infinitesimal gauge transformation.

\section{Preliminaries and Notation}

Throughout the paper the gauge group is assumed to be $\mathbf{U}(\imath), \imath \geqq 2$; and it is well known that it can always be reduced to $\mathbf{S U}(r)$ in the global case. Further, $r$ designates the rank of the holomorphic or instanton bundle under consideration 
and $c=c_{2}$ designates its topological charge (instanton number) equal to the second Chern class.

Let $\mathbb{C}^{4}$ be a 4-dimensional complex vector space with a fixed (standard) basis $\left\{e_{1}, e_{2}, e_{3}, e_{4}\right\}$ and, consequently, with the coordinates $z_{1}, z_{2}, z_{3}, z_{4}$, and also with the real structure

$$
\tau:\left(z_{1}, z_{2}, z_{3}, z_{4}\right) \mapsto\left(-\bar{z}_{2}, \bar{z}_{1},-\bar{z}_{4}, \bar{z}_{3}\right) .
$$

This real structure is transferred to the projective space $\mathbb{P}^{3}=\mathbb{P}\left(\mathbb{C}^{4}\right)$ as an antiholomorphic involution denoted again by $\tau$. In what follows, all $\tau$-invariant objects are referred to as real.

The manifold of real lines in $\mathbb{P}^{3}$ is the sphere $\mathbf{S}^{4}$. Since each point in $\mathbb{P}^{3}$ lies on the unique real line, we have the projection $\pi: \mathbb{P}^{3} \rightarrow \mathbf{S}^{4}$, the so-called Penrose transformation. Let $\mathbb{G}_{2}=\mathbb{G}_{2}\left(\mathbb{C}^{4}\right)$ be the Grassmann manifold points of which are lines in $\mathbb{P}^{3}$, and $\mathbb{F}_{1,2}=\mathbb{F}_{1,2}\left(\mathbb{C}^{4}\right)$ be the flag manifold points of which are the pairs $(Q, \mathscr{L}) \in \mathbb{P}^{3} \times \mathbb{G}_{2}, Q \in \mathscr{L}$. The real structure $\tau$ is again naturally transferred to $\mathbb{G}_{2}$ and to $\mathbb{F}_{1,2}$. Relate to each point from $\mathbb{P}^{3}$ the unique real line containing it to get an embedding of $\mathbb{P}^{3}$ into $\mathbb{F}_{1,2}$. The sphere as the manifold of real lines in $\mathbb{P}^{3}$ is embedded into $\mathbb{G}_{2}$; it is a real submanifold of $\mathbb{G}_{2}$ consisting of the real ( $\tau$-invariant) points. Summing up, we have the following standard commutative diagram, the embedding are real analytic:

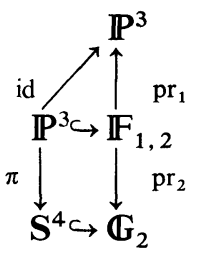

Denote by $\mathbb{P}^{2}$ the two-dimensional projective space embedded into $\mathbb{G}^{2}$ and consisting of those lines in $\mathbb{P}^{3}$ which contain the point $P_{0}=\operatorname{span} e_{4}$. Clearly, $\mathbf{S}^{4} \cap \mathbb{P}^{2}=\left\{x_{0}\right\}$ is a one-point set. The manifold $\operatorname{pr}_{2}^{-1}\left(\mathbb{P}^{2}\right) \subset \mathbb{F}_{1,2}$ is the blow-up of $\mathbb{P}^{3}$ at the point $P_{0}$ and it will be denoted by $\tilde{\mathbb{P}}^{3}$.

We distinguish the following objects in $\mathbb{P}^{3}$ : the points

the real lines

$$
P_{0}=\operatorname{span} e_{4}, \quad P_{\infty}=\operatorname{span} e_{3},
$$

the planes

$$
\mathscr{L}_{0}=\mathbb{P}\left(\left(e_{3}, e_{4}\right)\right)=\overline{P_{0} P_{\infty}}, \quad \mathscr{L}_{\infty}=\mathbb{P}\left(\left(e_{1}, e_{2}\right)\right),
$$

$$
H_{\infty}=\mathbb{P}\left(\left(e_{1}, e_{2}, e_{3}\right)\right), \quad H_{0}=\mathbb{P}\left(\left(e_{1}, e_{3}, e_{4}\right)\right) .
$$

The real lines $\mathscr{L}_{0}, \mathscr{L}_{\infty}$, if considered as points in $\mathbf{S}^{4}$, will be denoted by $x_{0}, x_{\infty}$ (or 0 , $\infty)$, respectively.

The restriction $\pi: H_{\infty} \backslash \mathscr{L}_{\infty} \rightarrow \mathbf{S}^{\mathbf{4}} \backslash\{\infty\}$ is one-to-one and it induces a complex structure on $\mathbf{S}^{4} \backslash\{\infty\}$ which we shall regard as the standard one. We choose the complex coordinates $y$, $z$ on $\mathbf{S}^{4} \backslash\{\infty\} \cong \mathbb{C}^{2}$ via the identification

$$
H_{\infty} \ni \operatorname{span}\left(y e_{1}+z e_{2}+e_{3}\right) \mapsto(y, z) \in \mathbb{C}^{2},
$$

and we introduce the coordinates

$$
\xi=z_{1} / z_{4}, \quad \eta=z_{2} / z_{4}, \quad \zeta=z_{3} / z_{4}
$$

on $\mathbb{P}^{3} \backslash H_{\infty}$. Since for each point $Q \in \mathbb{P}^{3} \backslash \mathscr{L}_{\infty}$ the plane $\overline{\mathscr{L}_{\infty} Q}$ intersects the line $\mathscr{L}_{0}$ in the unique point, we have the real analytic diffeomorphism

$$
\mathbb{P}^{3} \backslash H_{\infty} \cong \mathbb{C}^{3} \rightarrow\left(\mathbf{S}^{4} \backslash\{\infty\}\right) \times\left(\mathscr{L}_{0} \backslash\left\{P_{\infty}\right\}\right) \cong \mathbb{C}^{3} .
$$


Choosing the coordinate $\lambda=z_{3} / z_{4}$ on $\mathscr{L}_{0}$, we have the transformations

$$
y=(\xi \bar{\zeta}+\bar{\eta}) /(1+\zeta \bar{\zeta}), \quad z=(\eta \bar{\zeta}-\bar{\xi}) /(1+\zeta \bar{\zeta}), \quad \lambda=\zeta
$$

and

$$
\xi=\lambda y-\bar{z}, \quad \eta=\lambda z+\bar{y} .
$$

So one can express the functions on $\mathbb{C}^{3}$ in the coordinates $y, z, \lambda$ or $\xi, \eta, \zeta$. Provided the latter ones are used, the corresponding function will be underlined.

According to the fundamental theorem due to Atiyah, Hitchin and Singer [4], one can relate to every self-dual gauge field defined on a neighborhood $\mathscr{U}$ of the origin $x_{0} \in \mathbf{S}^{4}$ a holomorphic rank- $\iota$ vector bundle $F$ on $\pi^{-1}(\mathscr{U})$, holomorphically trivial on all real lines $\mathscr{L}_{x}, x \in \mathscr{U}$, and with the additional structure-a holomorphic isomorphism $\sigma: \tau^{*} \bar{F} \rightarrow F^{*}$ inducing a positively definite Hermitian structure in the space of holomorphic sections $H^{0}\left(\mathscr{L}_{x}, F \mid \mathscr{L}_{x}\right)$, again for all $x \in \mathscr{U}$. Moreover, this correspondence is invertible and one-to-one, up to equivalence.

We shall restrict ourselves to the framed instanton bundles with a distinguished orthonormal frame on the line $\mathscr{L}_{0}$. The framed instanton moduli space will be denoted by $M(r, c)$ and $\mathcal{O} M(r, c)$ designates the moduli space of framed holomorphic bundles on $\mathbb{P}^{2}(\mathbb{C})$. The restriction from $\mathbb{P}^{3}$ to $H_{0} \cong \mathbb{P}^{2}$ induces a natural one-to-one mapping $M(\imath, c) \rightarrow \mathcal{O} M(\imath, c)[11]$.

To any framed instanton bundle $F$ there corresponds a gauge equivalence class of germs of local transition functions. In more detail, let $\mathscr{U}$ be an open neighborhood of the origin $0 \in \mathbb{C}^{2}$. Then the set $\pi^{-1}(\mathscr{U})$ can be identified with $\mathscr{U} \times \mathscr{L}_{0}$ and the real structure takes the form $\tau:(x, \lambda) \mapsto(x,-1 / \bar{\lambda})$. Choose a holomorphic frame $\left\{\sigma_{1}, \ldots, \sigma_{2}\right\}$ defined on $\mathscr{U} \times \mathscr{H}_{+}$, with $\mathscr{H}_{+}$being an open neighborhood of the unit disc $\{\lambda ;|\lambda| \leqq 1\}$ in $\mathscr{L}_{0}$, in such a way that it coincides with the distinguished frame on $\mathscr{L}_{0} \cap\left(\mathscr{U} \times \mathscr{H}_{+}\right)$. Apply the isomorphism $\sigma^{-1}$ to the frame dual to $\left\{\sigma_{1}, \ldots, \sigma_{i}\right\}$ to get a holomorphic frame of the bundle $\tau^{*} \bar{F}$ on $\mathscr{U} \times \mathscr{H}_{+}$ and so a holomorphic frame $\left\{\hat{\sigma}_{1}, \ldots, \hat{\sigma}_{2}\right\}$ of the bundle $F$ on $\mathscr{U} \times \mathscr{H}_{-}, \mathscr{H}_{-}=\tau\left(\mathscr{H}_{+}\right)$. The desired transition function $G=\left(G_{j k}\right)$ is defined on $\mathscr{U} \times \mathscr{V}$, with $\mathscr{V}=\mathscr{H}_{+} \cap \mathscr{H}_{-}$ being a neighborhood of the unit circle in $\mathscr{L}_{0}$, by the relation $\mathscr{S}_{k}=\sum \hat{\jmath}_{j} G_{j k}$, and it has the properties:

(i) $G(0, \lambda)=1$,

(ii) $G(\xi, \eta, \zeta)$ is holomorphic,

(iii) $G(x,-1 / \bar{\lambda})^{\dagger}=G(x, \lambda)$.

On the contrary, every matrix valued function $G$ satisfying (i-iii) is a transition function of a local framed instanton bundle $F$. The distinguished trivialization on $\mathscr{L}_{0}$ is induced by the standard basis in $\mathbb{C}^{i}$.

The space of germs of local transition functions fulfilling (i-iii) will be denoted by $\overline{\mathfrak{G}}_{u}$. The above described correspondence can be established even if all reality conditions are omitted. This means that we do not insist on the existence of the isomorphism $\sigma$ as well as on the property (iii) of $G$, and, of course, the distinguished frame is not required to be orthonormal. The larger space of germs of local transition functions satisfying only the conditions (i), (ii) will be denoted by $\overline{\mathfrak{G}}$.

Let $\Gamma(x, \lambda)$ be a matrix valued function on $\mathscr{U} \times \mathscr{V}$ such that $\underline{\Gamma}(\xi, \eta, \zeta)$ is holomorphic and $\Gamma(0, \lambda)=1$. Then the corresponding gauge transformation takes the form

$$
G(x, \lambda) \mapsto \Gamma(x,-1 / \bar{\lambda})^{\dagger} G(x, \lambda) \Gamma(x, \lambda) .
$$

The objects we are dealing with are real analytic in some neighborhood of the origin in $\mathbb{C}^{2}$ and hence they can be locally extended from $\mathbb{C}^{2}$ to $\mathbb{C}^{4}$ as convergent power series in the variables $y, z, \bar{y}$, $\bar{z}$. In what follows, the symbol $x$ stands for four complex variables $y, z, \bar{y}, \bar{z}$ in this order. 
Let $\bar{\Xi}$ designate the subspace of the vector space

$$
\overline{\mathfrak{g}} \overline{\mathfrak{l}}(\iota, \mathbb{C} \llbracket x \rrbracket) \times \overline{\mathfrak{g}} \overline{\mathfrak{l}}(\iota, \mathbb{C} \llbracket x, \lambda \rrbracket) \times \overline{\mathfrak{g}} \overline{\mathfrak{l}}\left(\iota, \mathbb{C} \llbracket x, \lambda^{-1} \rrbracket\right)
$$

consisting of those matrices $(J, W, \hat{W})$ of formal power series which satisfy

$$
\begin{gathered}
W(x, 0)=\hat{W}(x, \infty)=\mathbf{1}, \\
J(0)=W(0, \lambda)=\hat{W}(0, \lambda)=\mathbf{1},
\end{gathered}
$$

and solve the equations

$$
\begin{gathered}
\partial_{z} W-\lambda J \partial_{\bar{y}}\left(J^{-1} W\right)=\partial_{y} W+\lambda J \partial_{\bar{x}}\left(J^{-1} W\right)=0, \\
\partial_{\bar{y}} \hat{W}-\lambda^{-1} J^{-1} \partial_{z}(J \hat{W})=\partial_{\bar{x}} \hat{W}+\lambda^{-1} J^{-1} \partial_{y}(J \hat{W})=0 .
\end{gathered}
$$

We shall write

$$
W(x, \lambda)=\mathbf{1}+\sum_{j=1}^{\infty} W_{j}(x) \lambda^{j}, \quad \hat{W}(x, \lambda)=\mathbf{1}+\sum_{j=1}^{\infty} \hat{W}_{j}(x) \lambda^{-j},
$$

where $W_{j}(x), \hat{W}_{j}(x) \in \overline{\mathfrak{g}} \overline{\mathrm{l}}(\iota, \mathbb{C} \llbracket x \rrbracket)$ and $W_{j}(0)=\hat{W}_{j}(0)=0$. An involution denoted again by $\sigma$ acts on $\overrightarrow{\mathbb{E}}$ :

$$
(J(x), W(x, \lambda), \hat{W}(x, \lambda)) \stackrel{\sigma}{\rightarrow}\left(J(x)^{\dagger},\left(\hat{W}(x,-1 / \bar{\lambda})^{\dagger}\right)^{-1},\left(W(x,-1 / \bar{\lambda})^{\dagger}\right)^{-1}\right) .
$$

We assume that the Hermitian conjugation includes the formal complex conjugation of the variables $y, z, \bar{y}, \bar{z}, \lambda, \bar{\lambda}$ (c.c. $y=\bar{y}$, c.c. $\bar{y}=y$, etc.). The $\sigma$-invariant subspace consisting of those $(J, W, \hat{W})$ which fulfil

$$
J(x)^{\dagger}=J(x), \quad \hat{W}(x, \lambda)^{-1}=W(x,-1 / \bar{\lambda})^{\dagger}
$$

will be denoted by $\widetilde{\mathfrak{S}}_{u}$.

The gauge transformations

$$
\begin{gathered}
J(x)^{-1} \mapsto \Gamma(x, 0)^{\dagger} J(x)^{-1} \Gamma(x, 0), \\
W(x, \lambda) \mapsto \Gamma(x, 0)^{-1} W(x, \lambda) \Gamma(x, \lambda)
\end{gathered}
$$

make sense on $\overline{\mathfrak{S}}_{u}$ provided $\Gamma \in \overline{\mathfrak{g}} \overline{\mathrm{I}}(\iota, \mathbb{C} \llbracket x, \lambda \rrbracket)$ satisfies

and

$$
\Gamma(0, \lambda)=\mathbf{1}
$$

$$
\left(\lambda \partial_{\bar{y}}-\partial_{x}\right) \Gamma=\left(\lambda \partial_{\bar{x}}+\partial_{y}\right) \Gamma=0 .
$$

The last condition means that

$$
\Gamma(x, \lambda)=\Gamma(\lambda y-\bar{z}, \lambda z+\bar{y}, \lambda)
$$

where $\underline{\Gamma}(\xi, \eta, \zeta) \in \overline{\mathrm{g}} \overline{\mathrm{I}}(\iota, \mathbb{C} \llbracket \xi, \eta, \zeta \rrbracket)$.

For $G \in \overline{\mathfrak{G}}$, we choose the Birkhoff decomposition in the form

$$
G(x, \lambda)=(J(x) \hat{W}(x, \lambda))^{-1} W(x, \lambda),
$$

where the matrix valued functions $W(x, \lambda)$ and $\hat{W}(x, \lambda)$ are holomorphic in $\lambda$ on neighborhoods of the discs $\{|\lambda| \leqq 1\}$ and $\left\{\left|\lambda^{-1}\right| \leqq 1\right\}$, respectively, provided $x$ is close enough to 0 , and they are normed by $W(x, 0)=\hat{W}(x, \infty)=1$. It is well known $[13,14]$ that in this way we get the embeddings $\overline{\mathscr{G}} \subset \overline{\mathfrak{S}}$ and $\overline{\mathfrak{F}}_{u} \subset \overline{\mathfrak{S}}_{u}$. In fact, this is the main link between the global approach and the local approach. 


\section{The Canonical Initial Condition}

One can exclude $J$ from (2.6), $J \partial_{\bar{y}} J^{-1}=\partial_{x} W_{1}, J \partial_{\bar{x}} J^{-1}=-\partial_{y} W_{1}$, to get

$$
\partial_{x} W-\lambda\left(\partial_{x} W_{1}\right) W-\lambda \partial_{\bar{y}} W=\partial_{y} W-\lambda\left(\partial_{y} W_{1}\right) W+\lambda \partial_{\bar{z}} W=0
$$

Takasaki's approach provides a method how to solve the equations (3.1) in the realm of formal power series, provided an initial condition

$$
W(y, z, 0,0, \lambda)=W^{(0)}(y, z, \lambda)
$$

is given. In accordance with (2.4), (2.5), the initial condition $W^{(0)} \in \overline{\mathfrak{g}} \bar{l}(\iota, \mathbb{C} \llbracket y, z, \lambda \rrbracket)$ is required to fulfill

$$
W^{(0)}(y, z, 0)=W^{(0)}(0,0, \lambda)=1 .
$$

It is known [10] that the initial value problem has the unique solution $W(x, \lambda)$ unambiguously determined by the condition

$$
W(x, \lambda) W^{(0)}\left(y-\lambda^{-1} \bar{z}, z+\lambda^{-1} \bar{y}, \lambda\right)^{-1} \in \overline{\mathfrak{g}} \overline{\mathfrak{l}}\left(\iota, \mathbb{C} \llbracket x, \lambda^{-1} \rrbracket\right) .
$$

This means that the left-hand side does not contain positive powers of $\lambda$. We can complete this result.

\section{Proposition 3.1. It holds}

$$
W(x, \lambda) W^{(0)}\left(y-\lambda^{-1} \bar{z}, z+\lambda^{-1} \bar{y}, \lambda\right)^{-1}=J(x) \hat{W}(x, \lambda),
$$

where $\hat{W}$ is normed by $\hat{W}(x, \infty)=1$ and $(J, W, \hat{W})$ solve (2.6), (2.7), and (2.5), i.e., $(J, W, \hat{W}) \in \bar{\Xi}$.

Proof. In fact, we shall prove also Takasaki's result in an alternative way. Put

$$
H(x, \lambda):=W^{(0)}\left(y-\lambda^{-1} \bar{z}, z+\lambda^{-1} \bar{y}, \lambda\right)^{-1} \in \overline{\mathfrak{g}} \bar{l}\left(\imath, \mathbb{C}\left[x, \lambda, \lambda^{-1} \rrbracket\right) .\right.
$$

Then $H(0, \lambda)=1$ and the $m^{\text {th }}$ homogeneous term $H_{m}$, in the variables $x$ does not contain powers of $\lambda$ lower than $\lambda^{-m}$. Consequently, $H(x, \lambda)$ is well defined. Moreover, the following assertion is valid:

For any $R \in \overline{\mathrm{g}} \overline{\mathrm{I}}(\iota, \mathbb{C} \llbracket x \rrbracket)$ there exists the unique solution $X \in \overline{\mathrm{g}} \overline{\mathrm{I}}(\imath, \mathbb{C} \llbracket x, \lambda \rrbracket)$ to the following problem:

$$
\begin{gathered}
X(x, \lambda) H(x, \lambda) \in \overline{\mathfrak{g}} \overline{\mathrm{I}}\left(\iota, \mathbb{C}\left[x, \lambda^{-1}\right]\right), \\
X(0, \lambda)=R(0) \text { and } X(x, 0)=R(x) .
\end{gathered}
$$

Actually, write $X$ in the form

$$
X(x, \lambda)=R(0)+\sum_{k=1}^{\infty} X_{k}(x, \lambda),
$$

with the terms $X_{k}$, being $k$-homogeneous in the variables $x$, to get the relations

$$
X_{k}+\sum_{j=1}^{k} H_{j}, X_{k-j}, \in \overline{\mathfrak{g}} \bar{l}\left(\imath, \mathbb{C}\left[x, \lambda^{-1}\right]\right), \quad k \geqq 1,
$$

which enable one to compute recursively and together with the condition $X(x, 0)$ $=R(x)$ unambiguously all terms $X_{k}$.

Now we can finish the proof. The condition (3.4) together with $W(0, \lambda)$ $=W(x, 0)=1$ has the unique solution $W$ and it can be easily seen that this $W$ also fulfills the initial condition (3.2). Now decompose

$$
W(x, \lambda) H(x, \lambda)=J(x) \hat{W}(x, \lambda),
$$


with the normalization $\hat{W}(x, \infty)=\mathbf{1}$. Clearly,

and hence

$$
\left(\partial_{z}-\lambda \partial_{\bar{y}}\right) H=\left(\partial_{y}+\lambda \partial_{\bar{z}}\right) H=0,
$$

$$
\left(\partial_{x}-\lambda \partial_{\bar{y}}\right)\left(J^{-1}(x) W(x, \lambda)\right) H(x, \lambda)=\left(\partial_{x}-\lambda \partial_{\bar{y}}\right) \hat{W}(x, \lambda) \in \overline{\mathfrak{g}} \overline{\mathbb{I}}\left(\iota, \mathbb{C}\left[x, \lambda^{-1} \rrbracket\right) .\right.
$$

At the same time, it holds

and

$$
\left.\left(\partial_{x}-\lambda \partial_{\bar{y}}\right)\left(J^{-1}(x) W(x, \lambda)\right)\right|_{x=0}=\left.\partial_{x}\left(J^{-1}(x) W(x, 0)\right)\right|_{x=0}=\partial_{x} J^{-1}(0)
$$

$$
\left.\left(\partial_{x}-\lambda \partial_{\bar{y}}\right)\left(J^{-1}(x) W(x, \lambda)\right)\right|_{\lambda=0}=\partial_{x} J^{-1}(x) .
$$

But $\left(\partial_{x} J^{-1}(x)\right) W(x, \lambda)$ fulfills the same relations as $\left(\partial z-\lambda \partial_{\bar{y}}\right)\left(J^{-1}(x) W(x, \lambda)\right)$ and so, according to the above assertion, we have the equality

$$
\left(\partial_{x}-\lambda \partial_{\bar{y}}\right)\left(J^{-1}(x) W(x, \lambda)\right)=\left(\partial_{x} J^{-1}(x)\right) W(x, \lambda) .
$$

Analogously we get

$$
\left(\partial_{y}+\lambda \partial_{\bar{x}}\right)\left(J^{-1}(x) W(x, \lambda)\right)=\left(\partial_{y} J^{-1}(x)\right) W(x, \lambda),
$$

i.e., Eq. (2.6) are satisfied. Further,

$$
\left(\partial_{x}-\lambda \partial_{\bar{y}}\right) \hat{W}(x, \lambda)=\left(\partial_{x} J^{-1}(x)\right) W(x, \lambda) H(x, \lambda)=\left(\partial_{x} J^{-1}(x)\right) J(x) \hat{W}(x, \lambda),
$$

and analogously,

$$
\left(\partial_{y}+\lambda \partial_{\bar{z}}\right) \hat{W}(x, \lambda)=\left(\partial_{y} J^{-1}(x)\right) J(x) \hat{W}(x, \lambda) .
$$

Hence Eq. (2.7) are satisfied as well. The rest of the proof is evident.

As a rule, the gauge freedom in the inverse scattering approach is used to get the self-dual equations in the form (2.6), (2.7). But there still remains an infinitedimensional group of gauge transformations preserving these equations. We shall show that, owing to this fact, one can distinguish a special solution in each gauge equivalence class. On the other hand, in this way the gauge freedom is eliminated completely.

For $\Gamma \in \overline{\mathfrak{g}} \overline{\mathfrak{I}}(\imath, \mathbb{C} \llbracket x, \lambda \rrbracket)$ put $\Gamma^{(0)}(y, z, \lambda):=\Gamma(y, z, 0,0, \lambda)$, and suppose the conditions (2.10), (2.11), (2.12) to be valid. Then

$$
\Gamma^{(0)}(y, z, \lambda)=\underline{\Gamma}(\lambda y, \lambda z, \lambda)=1+\sum_{j=1}^{\infty} \Gamma_{j}^{(0)}(y, z) \lambda^{j} .
$$

The relation (3.6) has the following consequences: $\Gamma_{j}^{(0)}(y, z)$ is a polynomial of degree equal at most to $j$ and, since $\Gamma^{(0)}(0,0, \lambda)=\Gamma(0, \lambda)=1$, the absolute term is zero. Moreover, (3.6) implies that, knowing $\Gamma^{(0)}$, we are able to reconstruct $\underline{\Gamma}$ and hence $\Gamma$ as well.

Since $\Gamma^{(0)}(y, z, 0)=\Gamma(0,0,0)=1$ and according to (2.9), the gauge transformation of the initial condition takes the form

Again we put

$$
W^{(0)} \mapsto W^{(0)} \Gamma^{(0)} .
$$

$$
W^{(0)}(y, z, \lambda)=\mathbf{1}+\sum_{j=1}^{\infty} W_{j}^{(0)}(y, z) \lambda^{j}, \quad W_{j}^{(0)}(y, z) \in \overline{\mathfrak{g}} \overline{\mathfrak{l}}(\iota, \mathbb{C} \llbracket y, z \rrbracket),
$$

and according to $(3.3), W_{j}^{(0)}(0,0)=0$. The best we can do is to transform off all summands in $W_{j}^{(0)}$ with degrees less than or equal to $j$. Moreover, the relation (3.7) 
implies that $\Gamma^{(0)}$ with this property is unique and, according to the above discussion, $\Gamma$ is unique as well. Summing up, we claim that

For each $(J, W, \hat{W}) \in \bar{\Xi}_{u}$ there exists the unique gauge transformation $\Gamma_{c}$ such that $W^{(0)}$ having been transformed satisfies

$$
W_{j}^{(0)}(y, z) \in \overline{\mathrm{g}} \mathrm{T}\left(\iota,(y, z)^{j+1}\right), \quad j \in \mathbb{N},
$$

where $(y, z)^{j+1}$ is the $(1+j)^{\text {th }}$ power of the ideal $(y, z) \subset \mathbb{C} \llbracket y, z \rrbracket$ generated by $y, z ;$ in other words, the formal power series $W_{j}^{(0)}(y, z)$ does not contain summands of degrees less than or equal to $j$.

Moreover, due to the uniqueness, this condition eliminates the gauge freedom. The unique gauge transformation $\Gamma_{c}$ will be called canonical and we shall derive an explicit formula for it. We shall need the following

Lemma 3.2. Assume that $(J, W, \hat{W}) \in \bar{\Xi}$ and

Then

$$
J(y, z, 0,0)=\hat{W}(y, z, 0,0, \lambda)=\mathbf{1} .
$$

(i)

$$
\hat{W}_{k}(x) \in \overline{\mathfrak{g}} \overline{\mathfrak{l}}\left(\imath,(\bar{y}, \bar{z})^{k+1}\right), \quad k \geqq 1,
$$

where $(\bar{y}, \bar{z})$ is now an ideal in $\mathbb{C} \llbracket x \rrbracket$ generated by $\bar{y}, \bar{z}$, and

$$
W(x, \lambda) W^{(0)}\left(y-\lambda^{-1} \bar{z}, z+\lambda^{-1} \bar{y}, \lambda\right)^{-1}=J(x) \hat{W}(x, \lambda) .
$$

Proof. (i) We have to show $\partial_{\bar{y}}^{i} \partial_{\bar{z}}^{j} \hat{W}_{k}(y, z, 0,0)=0$ for $0 \leqq i+j \leqq k$. According to the assumption, this assertion is valid for $i=j=0$. The Eq. (2.7) are equivalent to

$$
\begin{gathered}
\partial_{\bar{y}} \hat{W}_{k}=\partial_{x} \hat{W}_{k-1}+\left(J^{-1} \partial_{x} J\right) \hat{W}_{k-1}, \\
\partial_{\bar{z}} \hat{W}_{k}=-\partial_{y} \hat{W}_{k-1}-\left(J^{-1} \partial_{y} J\right) \hat{W}_{k-1}, \quad k \geqq 1 .
\end{gathered}
$$

Put $\bar{y}=\bar{z}=0$ in these relations to verify the assertion for $k=1$. Further we can proceed by induction in $k$. It suffices to differentiate these equations by $\partial_{\bar{y}}^{i-1} \partial_{\bar{x}}^{j}$ or by $\partial_{\bar{y}}^{i} \partial_{\bar{x}}^{j-1}$, respectively.

(ii) According to Proposition 3.1, the left-hand side in (3.10) equals to $J^{\prime}(x) \hat{W}^{\prime}(x, \lambda)$ with $\left(J^{\prime}, W, W^{\prime}\right) \in \widetilde{\mathbb{E}}$. It is sufficient to show that the initial condition (3.9) determines $J, \hat{W}$ unambiguously. But this is an immediate consequence of the following two easily verifiable assertions:

Let $(J, W)$ and $\left(J^{\prime}, W\right)$ solve (2.6) and satisfy the corresponding boundary conditions in (2.4), (2.5). Then $J^{\prime}=J X$ with

$$
X \in \overline{\mathfrak{g}} \mathfrak{T}(i, \mathbb{C} \llbracket y, z \rrbracket), \quad X(0)=\mathbf{1} .
$$

Let $(J, \hat{W}),\left(J, \hat{W}^{\prime}\right)$ solve $(2.7)$ and satisfy the corresponding boundary conditions in (2.4), (2.5). Then

with

$$
\hat{W}^{\prime}(x, \lambda)=Y\left(y-\lambda^{-1} \bar{z}, z+\lambda^{-1} \bar{y}, \lambda\right) \hat{W}(x, \lambda)
$$

$$
Y(\xi, \eta, \zeta) \in \overline{\mathfrak{g}} \overline{\mathrm{T}}\left(\iota, \mathbb{C} \llbracket \xi, \eta, \zeta^{-1} \rrbracket\right), \quad Y(0,0, \zeta)=\mathbf{1} .
$$

The following theorem contains the main information. 
Theorem 3.3. Let $(J, W, \hat{W}) \in \overline{\mathfrak{S}}_{u}$ and $J, \hat{W}$ fulfill (3.9), i.e.,

$$
J(y, z, 0,0)=\hat{W}(y, z, 0,0, \lambda)=\mathbf{1} .
$$

Then $W^{(0)}$ satisfies (3.8), i.e.,

and, moreover,

$$
W_{j}^{(0)}(y, z) \in \overline{\mathfrak{g}} \bar{l}\left(\imath,(y, z)^{j+1}\right), \quad j \geqq 1,
$$

$$
W^{(0)}(\bar{\lambda} \bar{z},-\bar{\lambda} \bar{y},-1 / \bar{\lambda})^{\dagger}=W^{(0)}(y, z, \lambda) .
$$

On the contrary, let $W^{(0)}$ satisfy (3.8), (3.12). Then there exists the unique solution $(J, W, \hat{W}) \in \overline{\mathfrak{S}}_{u}$ with $W^{(0)}$ being the initial condition for $W$ and, moreover, this solution fulfills (3.9).

Remark. $W^{(0)}(\bar{\lambda} \bar{z},-\bar{\lambda} \bar{y},-1 / \bar{\lambda})$ makes sense owing to (3.8). The relation (3.12) restores the reality condition which was abandoned in Takasaki's approach.

Proof. $(\Rightarrow)$ The validity of (3.8) follows from Lemma 3.2.ad(i) and from the equality

$$
W^{(0)}(y, z, \lambda)^{-1}=\hat{W}(0,0, \bar{y}, \bar{z},-1 / \bar{\lambda})^{\dagger} .
$$

By the assumption, the equality (3.10) holds. Set $y=z=0$ in it and make use of (3.9), (2.8) to get

$$
W^{(0)}\left(-\lambda^{-1} \bar{z}, \lambda^{-1} \bar{y}, \lambda\right)^{-1}=\hat{W}(0,0, \bar{y}, \bar{z}, \lambda) .
$$

Now, to prove (3.12), it is sufficient to replace $\lambda$ by $-1 / \bar{\lambda}$ and to perform Hermitian conjugation and inversion of both sides in the last equality.

$(\Leftarrow)$ We relate to $W^{(0)}$ a solution $(J, W, \hat{W}) \in \widetilde{\Xi}^{-}$according to Proposition 3.1. From (3.12) it follows that

$$
W^{(0)}(y+\bar{\lambda} \bar{z}, z-\bar{\lambda} \bar{y},-1 / \bar{\lambda})^{\dagger}=W^{(0)}\left(y-\lambda^{-1} \bar{z}, z+\lambda^{-1} \bar{y}, \lambda\right) .
$$

The $k^{\text {th }}$ homogeneous term in the variables $x$ in $W^{(0)}\left(y-\lambda^{-1} \bar{z}, z+\lambda^{-1} \bar{y}, \lambda\right)$ contains powers in $\lambda$ not lower than $(-k+1)$ and not greater than $(k-1)$. Consequently, we are allowed to multiply Eq. (3.5) from the right by this expression. So we have

$$
J(x)^{-1} W(x, \lambda)=\hat{W}(x, \lambda) W^{(0)}\left(y-\lambda^{-1} \bar{z}, z+\lambda^{-1} \bar{y}, \lambda\right) .
$$

Replace $\lambda$ by $-1 / \bar{\lambda}$ and conjugate and invert both sides in this equality to find that $\left(\hat{W}(x,-1 / \bar{\lambda})^{\dagger}\right)^{-1}$ is another solution to the initial value problem with the initial condition $W^{(0)}$. By uniqueness, we have

and so

$$
\left(\hat{W}(x,-1 / \bar{\lambda})^{\dagger}\right)^{-1}=W(x, \lambda)
$$

$$
J(x)^{\dagger}\left(W(x,-1 / \bar{\lambda})^{\dagger}\right)^{-1}=J(x) \hat{W}(x, \lambda) .
$$

Hence $(J, W, \hat{W}) \in \mathbb{\Xi}_{u}$. Validity of (3.9) follows immediately from (3.5) and the uniqueness is guanranteed by Lemma 3.2.ad(ii).

Definition. A solution $(J, W, \hat{W}) \in \mathbb{\Xi}_{u}$ will be called canonical provided it fulfills (3.9). The subspace of canonical solutions will be denoted by $\mathbb{\mathfrak { \Xi }}_{c}$. An initial condition $W^{(0)}$ will be called canonical provided it fulfills (3.8), (3.12). The space of canonical initial conditions will be denoted by $\overline{\mathfrak{B}}$.

Hence $W^{(0)} \in \overline{\mathfrak{B}}$ iff it holds

$$
W^{(0)}(y, z, \lambda)=1+\sum_{k=1}^{\infty} \sum_{n=k+1}^{\infty} \sum_{j=0}^{n} W_{n j k}^{(0)} y^{j} z^{n-j} \lambda^{k},
$$


where

$$
W_{n j k}^{(0)} \in \overline{\mathfrak{g}} \overline{\mathrm{I}}(i, \mathbb{C}), \quad W_{n j k}^{(0)}=(-1)^{n+j+k} W_{n, n-j, n-k}^{(0) \dagger} .
$$

Hence $\overline{\mathfrak{W}}$ can be identified with $\mathbb{R}^{\infty}$ since each point from $\overline{\mathfrak{W}}$ corresponds to a sequence of complex matrices $W_{n j k}^{(0)}, 0 \leqq j \leqq n, 1 \leqq k<n$, and the condition (3.13) simply excludes the superfluous real variables.

Suppose $(J, W, \hat{W}) \in \overline{\mathfrak{S}}_{u}$. We introduce a gauge transformation $\Gamma_{c}(x, \lambda)$ $=\Gamma_{-c}(\lambda y-\bar{z}, \lambda z+\bar{y}, \lambda)$, where

$$
\Gamma_{c}(\xi, \eta, \zeta)=W(0,0, \eta,-\xi, \zeta)^{-1} J(0,0, \eta,-\xi) .
$$

Having performed this gauge transformation, we get another solution $\left(J_{c}, W_{c}, \hat{W}_{c}\right) \in \overline{\mathfrak{S}}_{u}$. From (2.9) it follows that $J_{c}, \hat{W}_{c}$ satisfy (3.9) and, according to Theorem 3.3, the initial condition $W_{c}^{(0)}$ fulfills (3.8). Hence

The relation (3.14) represents the announced explicit form of the canonical gauge transformation.

This means that we have just constructed the projection

$$
\mathrm{pr}_{c}: \overline{\mathfrak{\Xi}}_{u} \rightarrow \overline{\mathfrak{\Xi}}_{c}:(J, W, \hat{W}) \mapsto\left(J_{c}, W_{c}, \hat{W}_{c}\right),
$$

which, moreover, induces a one-to-one mapping of the quotient $\overline{\mathfrak{\Xi}}_{u} /$ gauge transformations onto $\overline{\mathfrak{S}}_{c}$. According to (3.9), (2.8), $\Gamma_{c}(\xi, \eta, \zeta)=\mathbf{1}$ provided $(J, W, \hat{W}) \in \widehat{\Xi}_{c}$ and hence the projection $\mathrm{pr}_{c}$ reduces to the identity mapping on $\widetilde{\mathfrak{S}}_{c} \subset \overline{\mathfrak{S}}_{u}$. According to Theorem 3.3, the mapping $\overline{\mathfrak{S}}_{c} \rightarrow \overline{\mathfrak{W}}:(J, W, \hat{W}) \mapsto W^{(0)}$ is oneto-one and so we have

$$
\overline{\mathfrak{S}}_{u} / \text { gauge transformations } \cong \overline{\mathfrak{S}}_{c} \cong \overline{\mathfrak{M}} \cong \mathbb{R}^{\infty} \text {. }
$$

In other words, the gauge equivalence classes of local self-dual solutions are parameterized by the points from the space $\overline{\mathfrak{B}} \cong \mathbb{R}^{\infty}$.

Denote by $\overline{\mathfrak{F}}_{c} \subset \overline{\mathfrak{F}}_{u}$ for the subspace of germs of those transition functions $G=(J, \hat{W})^{-1} W$ for which

$$
G(y, z, 0,0, \lambda)=W^{(0)}(y, z, \lambda) .
$$

By restriction, we obtain the projection

where

$$
\operatorname{pr}_{c}: \overline{\mathfrak{G}}_{u} \rightarrow \overline{\mathfrak{G}}_{c}: G=(J, \hat{W})^{-1} W \mapsto G_{c},
$$

$$
\underline{G}_{c}(\xi, \eta, \zeta)=W^{(0)}(\xi / \zeta, \eta / \zeta, \zeta) \hat{W}(\bar{\eta},-\bar{\xi}, 0,0,-1 / \bar{\zeta})^{\dagger} J(\bar{\eta},-\bar{\xi}, 0,0)^{\dagger}
$$

Again the projection $\mathrm{pr}_{c}$ induces a one-to-one mapping

$$
\overline{\mathfrak{G}}_{u} / \text { gauge transformations } \cong \overline{\mathfrak{G}}_{c} \text {. }
$$

Besides, one has a simple relation between the canonical transition function $G_{c}$ and the canonical initial condition $W_{c}^{(0)}$ :

$$
\begin{gathered}
G_{c}(\xi, \eta, \zeta)=W_{c}^{(0)}(\xi / \zeta, \eta / \zeta, \zeta), \quad \text { i.e., } \\
G_{c}(x, \lambda)=W_{c}^{(0)}\left(y-\lambda^{-1} \bar{z}, z+\lambda^{-1} \bar{y}, \lambda\right),
\end{gathered}
$$

and vice versa

$$
W_{c}^{(0)}(y, z, \lambda)=\underline{G}_{c}(\lambda y, \lambda z, \lambda) .
$$

The following proposition suggests that these results can have an interesting application also in the global case. 
Proposition 3.4. The isomorphism class of a global framed instanton bundle $F$ on $\mathbb{P}^{3}$ is unambiguously determined by its local restriction to $\pi^{-1}(\mathscr{U})$ with $\mathscr{U}$ being any open neighborhood of the origin $x_{0} \in \mathbf{S}^{4}$.

Proof. The Gram matrix of the Hermitian form, if expressed in a holomorphic frame, is real analytic. Owing to this fact, the distinguished orthonormal frame on $\mathscr{L}_{0}$ can be extended as an orthonormal real analytic trivialization $\left\{t_{1}, \ldots, t_{t}\right\}$ of $F$ over $\mathbb{P}^{\mathbf{3}} \backslash \mathscr{L}_{\infty}$ in the following way. The sections $t_{j}$ are defined on the plane $H_{\infty} \backslash \mathscr{L}_{\infty}$ $\cong \mathbb{C}^{2}$ as horizontal lifts over the segments $\overline{P_{\infty}} Q, Q \in H_{\infty} \backslash \mathscr{L}_{\infty}$, and they are extended as global holomorphic sections over each real line. Using this trivialization, we get a connection form $\mathscr{A}$ on $\mathbb{C}^{2} \cong \mathbb{R}^{4}$ with the curvature being self-dual and with the finite topological charge equal to $c_{2}(F)$. At the same time, $\mathscr{A}$ depends only on the isomorphism class of $F$ and is real analytic and hence unambiguously determined by its germ at the origin. Using now Uhlenbeck Theorem [15] to remove the singularity at $\infty$ and applying the Penrose twistor transformation, we conclude that $\mathscr{A}$ determines $F$ uniquely up to isomorphism.

It follows that, having in mind (3.16), (3.19), we can relate to each isomorphism class a unique point from the space $\overline{\mathfrak{B}}$. In this way we get an embedding of the framed instanton moduli space $M(\imath, c)$ into $\overline{\mathfrak{B}} \cong \mathbb{R}^{\infty}$. The explicit expressions will be given in Chap. 5 .

\section{The Geometric Interpretation}

Despite the local way of its definition, the canonical initial condition can be shown to have a clear geometric interpretation in the twistor framework. The global embedding of $S^{4}$ into $\mathbb{G}_{2}$ and the pull-back of the instanton bundle pri ${ }_{1}^{*} F$ on $\mathbb{F}_{1,2}$ corresponds to the local analytic extension from $\mathbb{C}^{2}$ to $\mathbb{C}^{4}$. The initial condition, i.e., the local restriction to the two-dimensional subspace $\mathbb{C}^{2} \subset \mathbb{C}^{4}$ determined by the equations $\bar{y}=\bar{z}=0$, has a counterpart on the global level in the restriction from $\mathbb{G}_{2}$ to $\mathbb{P}^{2}$ and from $\mathbb{F}_{1,2}$ to $\mathbb{P}^{3}$. Actually, in the local coordinates $y, z, \bar{y}, \bar{z}$ on $\mathbb{G}_{2}$ at the point $x_{0}$ introduced via the mapping

it holds:

$$
(y, z, \bar{y}, \bar{z}) \mapsto \mathbb{P}\left(y e_{1}+z e_{2}+e_{3},-\bar{z} e_{1}+\bar{y} e_{2}+e_{4}\right),
$$

$\mathrm{S}^{4}$ is locally determined by the equations

$$
\bar{y}=\text { c.c. } y, \quad \bar{z}=\text { c.c. } z,
$$

and $\mathbb{P}^{2}$ is locally determined by the equations

$$
\bar{y}=\bar{z}=0 \text {. }
$$

The main goal of this chapter is to describe a geometric construction of the canonical transition function. This construction and its consequences can be formulated in a more general setting.

Notation. Let us consider the $n$-dimensional projective space $\mathbb{P}^{n}, n \geqq 2$, with a fixed line $\mathscr{L}_{0}$ and a framed holomorphic rank- $r$ vector bundle $F$ on $\mathbb{P}^{n}, \imath \geqq 2$, with a distinguished holomorphic trivialization on $\mathscr{L}_{0}$. For such vector bundles, the first Chern class $c_{1}(F)$ vanishes and, according to Grothendieck's Theorem, the vector bundle decomposes on each line $\mathscr{L} \subset \mathbb{P}^{n}$ as a direct sum of line-bundles:

$$
F \mid \mathscr{L}=\mathcal{O}\left(i_{1}\right) \oplus \ldots \oplus \mathcal{O}\left(i_{2}\right), i_{1} \geqq \ldots \geqq i_{\imath}, i_{1}+\ldots+i_{\imath}=0 .
$$


Hence $i_{1} \geqq 0$ and $i_{1}=0$ iff $F$ is holomorphically trivial on $\mathscr{L}$. The set $\mathscr{S}_{F}$ of jumping lines in $\mathbb{P}^{n}$, i.e., consisting of those lines on which $F$ is not holomorphically trivial, is a proper closed analytic subset in the Grassmann manifold $\mathbb{G}_{2}\left(\mathbb{C}^{n+1}\right.$ ) (cf. [16], Chap. I). Fix two different points $P_{0}, P_{\infty} \in \mathscr{L}_{0}$ and denote by $\mathscr{S}_{0}, \mathscr{S}_{\infty}$ the sets of jumping lines passing through $P_{0}$ and $P_{\infty}$, respectively. Let $\operatorname{Sing}\left(P_{0}\right) \subset \mathbb{P}^{n}$ be the union of all lines belonging to $\mathscr{S}_{0}$, analogously define $\operatorname{Sing}\left(P_{\infty}\right)$. The set consisting of those lines in $\mathbb{P}^{n}$ which contain the point $P_{0}$ (respectively $P_{\infty}$ ) can be identified with the $(n-1)$-dimensional projective space and its subset $\mathscr{S}_{0}$ (respectively $\left.\mathscr{S}_{\infty}\right)$ is then proper closed and analytic. Consequently, $\operatorname{Sing}\left(P_{0}\right)$ and $\operatorname{Sing}\left(P_{\infty}\right)$ are proper closed analytic subsets in $\mathbb{P}^{n}$.

To get more information about jumping lines, we adapt the following theorem due to Barth by generalizing it to higher ranks. Denote by $\mathscr{F}$ the locally free rank- $\iota$ sheaf of germs of holomorphic sections in $F$. Recall the standard diagram:

$$
\begin{aligned}
& \mathfrak{F r}_{1,2} \stackrel{\mathrm{pr}_{1}=p}{\longrightarrow} \mathbb{P}^{n} \\
& \mathbb{G}_{2}
\end{aligned}
$$

Theorem 4.1. The set $\mathscr{S}_{F}$ of jumping lines is an analytic subset in the Grassmann manifold $\mathbb{G}_{2}$ of codimension one everywhere. The sheaf $\mathscr{E}:=R^{1} q_{*} p_{*}(\mathscr{F}(-1))($ the first direct image of the pulled-back sheaf $\left.p^{*}(\mathscr{F}(-1))\right)$ determines in $\mathbb{G}_{2}$ a divisor $\mathscr{D}_{F}$ the degree of which is equal to $c_{2}(F)$ and the support of which coincides with $\mathscr{S}_{F}$, $\operatorname{supp} \mathscr{D}_{F}=\operatorname{supp} \mathscr{E}=\mathscr{S}_{F}$.

Proof. In [16], Chap. II and 2.3, there is given a proof for the case $\imath=\operatorname{rank} F=2$. The main part of this proof can be reproduced almost verbatim also for higher ranks. We shall not do this and notice only the last part in which the degree of the divisor is computed. Hence we assume the following facts having been proved: $=\mathscr{S}_{F}$.

The sheaf $\mathscr{E}$ determines unambiguously a divisor $\mathscr{D}_{F}$ in $\mathbb{G}_{2}$ and $\operatorname{supp} \mathscr{D}_{F}=\operatorname{supp} \mathscr{E}$

It is sufficient to verify the equality $\operatorname{deg} \mathscr{D}_{\mathrm{F}}=c_{2}(F)$ only for the dimension $n=2$ (and that will be assumed up to the end of the proof).

In this case, there exists a resolution

with $k_{i}, m_{j}<0$.

$$
0 \rightarrow \bigoplus_{i=1}^{\stackrel{O}{O}} \mathcal{O}\left(k_{i}\right) \rightarrow \bigoplus_{j=1}^{t} \mathcal{O}\left(m_{j}\right) \rightarrow \mathscr{F}(-1) \rightarrow 0
$$

The line bundle corresponding to the divisor $\mathscr{D}_{F}$ is

where

$$
\left[\mathscr{D}_{F}\right]=\operatorname{det} E_{2} \otimes \operatorname{det} E_{1}^{*},
$$

$$
E_{1}=\oplus R^{1} q_{*} p^{*} \mathcal{O}\left(k_{i}\right), \quad E_{2}=\oplus R^{1} q_{*} p^{*} \mathcal{O}\left(m_{j}\right) .
$$

Finally, $c_{1}\left(R^{1} q_{*} p^{*} \mathcal{O}\left(k_{i}\right)\right)=-k(k+1) / 2$.

Now we can apply the Whitney formula to the resolution (4.1): $c_{1}(F)=0$, and hence

$$
\begin{aligned}
& c_{1}(F(-1))=-\imath=c_{1}\left(\oplus \mathcal{O}\left(m_{j}\right)\right)-c_{1}\left(\oplus \mathcal{O}\left(k_{i}\right)\right)=\sum m_{j}-\sum k_{i}, \\
c_{2}(F(-1))= & c_{2}(F)+\frac{1}{2} \imath(\imath-1)=c_{2}\left(\oplus \mathcal{O}\left(m_{j}\right)\right) \\
& -c_{2}\left(\oplus \mathcal{O}\left(k_{i}\right)\right)-c_{1}\left(\oplus \mathcal{O}\left(k_{i}\right)\right) c_{1}(F(-1)) \\
= & \sum_{i<j} m_{i} m_{j}-\sum_{i<j} k_{i} k_{j}+\imath \sum k_{i} .
\end{aligned}
$$


Finally, we can compute

$$
\begin{aligned}
\operatorname{deg} \mathscr{D}_{F}= & c_{1}\left(\left[\mathscr{D}_{F}\right]\right)=c_{1}\left(E_{2}\right)-c_{1}\left(E_{1}\right) \\
= & \frac{1}{2} \sum\left(k_{i}^{2}+k_{i}\right)-\frac{1}{2} \sum\left(m_{j}^{2}+m_{j}\right) \\
= & -\sum_{i<j} k_{i} k_{j}+\sum_{i<j} m_{i} m_{j}+\frac{1}{2}\left(\sum k_{i}\right)^{2} \\
& +\frac{1}{2} \sum k_{i}-\frac{1}{2}\left(\sum m_{j}\right)^{2}-\frac{1}{2} \sum m_{j} \\
= & c_{2}(F(-1))+\frac{1}{2} \imath\left(-\sum k_{i}+\sum m_{j}+1\right) \\
= & c_{2}(F(-1))-\frac{1}{2} \imath(\imath-1)=c_{2}(F) . \quad \square
\end{aligned}
$$

Corollary 4.2. Let $\mathbb{P}^{n} \subset \mathbb{P}^{n} \times \mathbb{P}^{n-1}$ be the blow-up of $\mathbb{P}^{n}$ at the point $P_{0} . \mathbb{P}^{n-1}$ is here considered as a submanifold in $\mathbb{G}_{2}$ consisting of those lines in $\mathbb{P}^{n}$ which contain the point $P_{0}$. Then $\mathscr{S}_{0}:=\mathscr{S}_{F} \cap \mathbb{P}^{n-1}$ is an analytic subset in $\mathbb{P}^{n-1}$ of codimension one everywhere and the sheaf $\mathscr{E}_{0}:=R^{1} \mathrm{pr}_{2 *} \operatorname{pr}_{1}^{*}(\mathscr{F}(-1))$ determines in $\mathbb{P}^{n-1}$ a divisor $\mathscr{D}_{0}$ of degree $c_{2}(F)$ and such that $\mathscr{S}_{0}=\operatorname{supp} \mathscr{E}_{0}=\operatorname{supp} \mathscr{D}_{0}$.

Proof. Since $\mathscr{S}_{F} \subset \mathbb{G}_{2}$ is of codimension one everywhere, the codimension of $\mathscr{S}_{0}$ $=\mathscr{S}_{F} \cap \mathbb{P}^{n-1}$ is at most one. But $\mathscr{S}_{0}$ is a proper closed analytic subset in $\mathbb{P}^{n-1}$, and hence it is of codimension one everywhere, too. Since supp $\mathscr{D}_{F}=\mathscr{S}_{F}, \mathscr{D}_{F}$ cuts in $\mathbb{P}^{n-1}$ a divisor $\mathscr{D}_{0}$ such that

and

$$
\operatorname{supp} \mathscr{D}_{0}=\left(\operatorname{supp} \mathscr{D}_{F}\right) \cap \mathbb{P}^{n-1}=\mathscr{S}_{0}, \quad\left[\mathscr{D}_{0}\right]=\left[\mathscr{D}_{F}\right] \mid \mathbb{P}^{n-1}
$$

$$
\operatorname{deg} \mathscr{D}_{0}=c_{1}\left(\left[\mathscr{D}_{0}\right]\right)=c_{1}\left(\left[\mathscr{D}_{F}\right]\right)=\operatorname{deg} \mathscr{D}_{F}=c_{2}(F) .
$$

Now one can make use of the following diagram

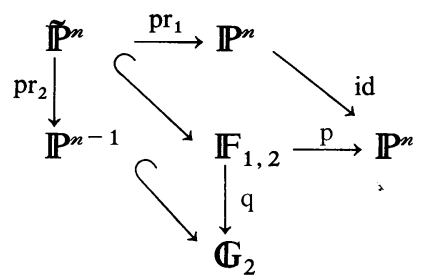

and apply the base-change theorem (cf. [17], Sect. 9) to get $\mathscr{E}_{0}=\mathscr{E} \mid \mathbb{P}^{n-1}$ and consequently $\operatorname{supp} \mathscr{E}_{0}=(\operatorname{supp} \mathscr{E}) \cap \mathbb{P}^{n-1}=\mathscr{S}_{0}$. Hence the divisor $\mathscr{D}_{0}$ is unambiguously determined by the sheaf $\mathscr{E}_{0}$.

Corollary 4.2 takes into account also the multiplicity of the irreducible components of the algebraic set $\mathscr{S}_{0}$. Let $\widetilde{\mathscr{D}}_{0}:=\operatorname{pr}_{1}\left(\operatorname{pr}_{2}{ }_{2}^{-1}\left(\mathscr{D}_{0}\right)\right)$ be the divisor in $\mathbb{P}^{n}$ (see (4.2)). Then $\operatorname{deg} \mathscr{D}_{0}=c_{2}(F)$ and $\operatorname{supp} \mathscr{D}_{0}=\operatorname{Sing}\left(P_{0}\right)$. Define analogously $\mathscr{D}_{\infty}$. Again, $\operatorname{deg} \widetilde{\mathscr{D}}_{\infty}=c_{2}(F)$ and $\operatorname{supp} \widetilde{\mathscr{D}}_{\infty}=\operatorname{Sing}\left(P_{\infty}\right)$.

Construction. Owing to the distinguished frame on $\mathscr{L}_{0}$, we have a fixed basis in the fibre over the point $P_{\infty}$. Extend this basis as a holomorphic trivialization on each line containing $P_{\infty}$ and not belonging to $\mathscr{S}_{\infty}$. We get a holomorphic frame $\left\{\diamond_{1}, \ldots, \diamond_{\imath}\right\}$ defined on the open set $\mathscr{U}_{0}=\mathbb{P}^{n} \backslash \operatorname{Sing}\left(P_{\infty}\right) \ni P_{0}$. Construct analogously the holomorphic frame $\left\{\tilde{\sigma}_{1}, \ldots, \hat{\jmath}_{\imath}\right\}$ on the open set $\mathscr{U}_{\infty}=\mathbb{P}^{n} \backslash \operatorname{Sing}\left(P_{0}\right) \ni P_{\infty}$. The corresponding transition fucntion is defined on $\mathscr{U}_{0} \cap \mathscr{U}_{\infty}=\mathbb{P}^{n} \backslash\left(\operatorname{Sing}\left(P_{0}\right)\right.$ $\left.\cup \operatorname{Sing}\left(P_{\infty}\right)\right)$ and it will be denoted by $G^{F}=\left(G_{j k}^{F}\right) ; \diamond_{k}=\sum \hat{\jmath}_{j} G_{j k}^{F}$.

According to Chow's Theorem, all the sets $\mathscr{S}_{0}, \mathscr{S}_{\infty}, \operatorname{Sing}\left(P_{0}\right), \operatorname{Sing}\left(P_{\infty}\right)$ are algebraic varieties in the corresponding projective spaces, and owing to a 
generalization of the same theorem, $F$ is an algebraic vector bundle on $\mathbb{P}^{n}$ (cf. [18], Sect. 1.3). $G^{F}$ should be a matrix of meromorphic functions on $\mathbb{P}^{n}$ and hence, according to the same principles, a matrix of rational functions on $\mathbb{P}^{n}$ with the poles on the algebraic set $\operatorname{Sing}\left(P_{0}\right) \cup \operatorname{Sing}\left(P_{\infty}\right)$. By the construction, $G^{F}$ is identically equal to 1 on the line $\mathscr{L}_{0}$, and so it has the following form (in the homogeneous coordinates $\bar{z}=\left(z_{1}, \ldots, z_{n}\right)$ on $\left.\mathbb{P}^{n}\right)$ :

$$
G^{F}(\overline{\mathrm{z}})=1+\frac{1}{p_{0}(\overline{\mathrm{z}}) p_{\infty}(\overline{\mathrm{z}})} \mathscr{R}(\overline{\mathrm{z}})
$$

where $h_{0}, h_{\infty}$ are homogeneous polynomials and $\mathscr{R}(\overline{\mathfrak{z}})$ is a matrix of homogeneous polynomials with the degrees equal to $\operatorname{deg}\left(\mu_{0}\right)+\operatorname{deg}\left(\not_{\infty}\right)$. Further, $\mathscr{R}(\bar{z}) \equiv 0$ on the line $\mathscr{L}_{0}$ and the algebraic set $p_{0}(\overline{\mathrm{z}})=0$ (respectively $\not_{\infty}(\overline{\mathrm{z}})=0$ ) coincides with $\operatorname{Sing}\left(P_{0}\right)\left(\right.$ respectively $\left.\operatorname{Sing}\left(P_{\infty}\right)\right)$, up to multiplicities. Respecting Corollary 4.2, we conjecture:

The polynomials $p_{0}(\bar{\jmath}), p_{\infty}(\bar{\jmath})$ in (4.3) can be chosen in such a way that the corresponding divisors $\left(\mu_{0}\right)$ and $\left(\mu_{\infty}\right)$ coincide with $\widetilde{\mathscr{D}}_{0}$ and $\widetilde{\mathscr{D}}_{\infty}$, respectively. Then $\operatorname{deg} \not_{0}=\operatorname{deg} \not_{\infty}=c_{2}(F)$.

The explicit results derived in the next chapter for instanton bundles support this conjecture.

$G^{F}$ can be shown to be unimodular.

Proposition 4.3. $\operatorname{det} G^{F}=1$.

Proof. The line bundle $\operatorname{det} F$ on $\mathbb{P}^{n}$ is holomorphically trivial. The choice of a frame in $F$ on the line $\mathscr{L}_{0}$ induces a holomorphic frame in $\operatorname{det} F$ on $\mathscr{L}_{0}$ which extends to a holomorphic trivialization over $\mathbb{P}^{n}$ in the unique way. From the construction of $G^{F}$ as a transition function it follows immediately that $\operatorname{det} G^{F}$ is a transition function of the bundlet $\operatorname{det} F$ and it is identically equal to 1 on $\mathscr{U}_{0} \cap \mathscr{U}_{\infty}$ and hence everywhere on $\mathbb{P}^{n}$.

By the construction, the matrix function $G^{F}$ depends only on the isomorphism class of the original framed vector bundle $F$. But the crucial property of $G^{F}$ is that it characterizes $F$ completely.

Theorem 4.4. The isomorphism class of a framed holomorphic vector bundle $F$ on $\mathbb{P}^{n}$ is unambiguously determined by the matrix valued function $G^{F}$.

Proof. $G^{F}$, if considered as a transition function, determines the framed vector bundle $F$ up to isomorphism over the open set $\mathscr{U}_{0} \cup \mathscr{U}_{\infty}=\mathbb{P}^{n} \backslash\left(\operatorname{Sing}\left(P_{0}\right) \cap \operatorname{Sing}\left(P_{\infty}\right)\right)$. The analytic sets $\operatorname{Sing}\left(P_{0}\right), \operatorname{Sing}\left(P_{\infty}\right)$ have dimensions equal to $(n-1)$ and their intersection has dimension equal at most to $(n-2)$.

Actually, provided the dimension of the intersection equals $(n-1)$, the analytic sets $\operatorname{Sing}\left(P_{0}\right), \operatorname{Sing}\left(P_{\infty}\right), \operatorname{Sing}\left(P_{0}\right) \cap \operatorname{Sing}\left(P_{\infty}\right)$ have the same dimensions and so they have at least one common irreducible component $\mathscr{T}$, again with dimension equal to $(n-1)$ (cf. [19], Sect. 1.3). The irreducible components of the analytic set $\operatorname{Sing}\left(P_{0}\right)$ are in one-to-one correspondence with the irreducible components of the analytic set $\mathscr{S}_{0}$ and each of them contains the point $P_{0}$. Consequently, $P_{0} \in \mathscr{T}$ $C \operatorname{Sing}\left(P_{\infty}\right)$ and we get a contradiction.

Now, let $2: \mathscr{U}_{0} \cup \mathscr{U}_{\infty} \hookrightarrow \mathbb{P}^{n}$ be the embedding. Owing to a consequence of Hartog's Theorem which guarantees the removability of singularities of a complex analytic function provided the singular points are contained in an analytic set of codimension at least two, we have a natural isomorphism $l_{*} l^{*} \mathscr{F} \cong \mathscr{F}$. Hence $F$ is determined by its restriction $F \mid\left(\mathscr{U}_{0} \cup \mathscr{U}_{\infty}\right)$ in the unique way. 
Let us now specify this construction for the framed instanton bundles on $\mathbb{P}^{3}$. This means that the real structure must be taken into account. Now, $\mathscr{L}_{0}$ is a real line, $\tau\left(P_{0}\right)=P_{\infty}$ and the distinguished frame on $\mathscr{L}_{0}$ is orthonormal. Moreover, $\tau\left(\operatorname{Sing}\left(P_{0}\right)\right)=\operatorname{Sing}\left(P_{\infty}\right)$ and the frame $\left\{\hat{\jmath}_{1}, \ldots, \hat{\jmath}_{2}\right\}$ is related to the dual of the frame $\left\{\sigma_{1}, \ldots, \diamond_{\imath}\right\}$ by the isomorphism $\sigma$. It follows that

$$
G^{F}(\tau(Q))=G^{F}(Q)^{\dagger} \quad \text { on } \quad \mathscr{U}_{0} \cap \mathscr{U}_{\infty} .
$$

The goal now is to show that $G^{F}$ can be identified with the locally defined canonical transition function $G_{c}$. First, using the blow-up we resolve the singularity at the point $P_{0}$ and then choose a special coordinate system at this point.

Denote by $\widetilde{P}_{0}=\left(P_{0}, \mathscr{L}_{0}\right)$ the point from the exceptional divisor in the blow-up $\mathbb{P}^{3}$. The pull-back of the matrix-valued function $\operatorname{pr}_{1}^{*} G^{F}$ is holomorphic at the point $\widetilde{P}_{0}$. Identify $\mathbb{P}^{2}$ with the plane $H_{\infty}=\mathbb{P}\left(e_{1}, e_{2}, e_{3}\right) \subset \mathbb{P}^{3}$ and introduce local coordinates on $\mathbb{P}^{3} \subset \mathbb{P}^{3} \times \mathbb{P}^{2}$ via the mapping

$$
(y, z, \lambda) \mapsto \operatorname{span}\left(\lambda y e_{1}+\lambda z e_{2}+\lambda e_{3}+e_{4}\right), \quad \operatorname{span}\left(y e_{1}+z e_{2}+e_{3}\right) ;
$$

the values in $\widetilde{P}_{0}$ are $y=z=\lambda=0$. Denote $\operatorname{pr}_{1}^{*} G^{F}$, if expressed in these local coordinates, by $W_{c}^{(0)}(y, z, \lambda)$. Clearly,

$$
W_{c}^{(0)}(y, z, 0)=W_{c}^{(0)}(0,0, \lambda)=1 .
$$

From (4.4) it follows that the equality

$$
W_{c}^{(0)}(\bar{\lambda} \bar{z},-\bar{\lambda} \bar{y},-1 / \bar{\lambda})^{\dagger}=W_{c}^{(0)}(y, z, \lambda)
$$

holds on an open set $\mathscr{U} \times \mathscr{V}$, where $\mathscr{U}$ and $\mathscr{V}$ are neighborhoods of the origin in $\mathbb{C}^{2}$ and of the unit circle in $\mathbb{C}$, respectively. Looking at the Lorain $\lambda$-expansion in this equality, we find that the condition (3.8) is valid and, consequently, the reality condition (3.12) is valid as well. Hence $W_{c}^{(0)}$ is indeed the canonical initial condition corresponding to the framed instanton bundle $F$.

The projection

$$
\operatorname{pr}_{1}: \mathbb{P}^{3} \rightarrow \mathbb{P}^{3}:\left(y^{\prime}, z^{\prime}, \lambda^{\prime}\right) \mapsto(\xi, \eta, \zeta)=\left(\lambda^{\prime} y^{\prime}, \lambda^{\prime} z^{\prime}, \lambda^{\prime}\right)
$$

is one-to-one provided $\lambda^{\prime} \neq 0$. For the real analytic coordinates $(x, \lambda) \equiv(y, z, \bar{y}, \bar{z}, \lambda)$ on $\mathbb{P}^{3}$, the relation $(2.2)$ implies:

and hence:

$$
\xi=\lambda y-\bar{z}=\lambda^{\prime} y^{\prime}, \quad \eta=\lambda z+\bar{y}=\lambda^{\prime} z^{\prime}, \quad \zeta=\lambda=\lambda^{\prime},
$$

$$
y^{\prime}=y-\lambda^{-1} \bar{z}, \quad z^{\prime}=z+\lambda^{-1} \bar{y}, \quad \lambda^{\prime}=\lambda .
$$

Since $W_{c}^{(0)}=\operatorname{pr}_{1}^{*} G^{F}$, we have

$$
G^{F}(x, \lambda)=W_{c}^{(0)}\left(y^{\prime}, z^{\prime}, \lambda^{\prime}\right)=W_{c}^{(0)}\left(y-\lambda^{-1} \bar{z}, z+\lambda^{-1} \bar{y}, \lambda\right) .
$$

Comparing this relation with (3.20), we conclude:

The matrix-valued function $G^{F}$, if expressed in the local coordinates $(x, \lambda)$ on $\mathbb{C}^{3} \cong \mathbb{P}^{3} \backslash H_{\infty}$, coincides with the canonical transition function $G_{c}$.

Note that this construction of the canonical transition function is applicable also to the local framed instanton bundles. 


\section{Explicit Expressions}

The global instanton solutions are described by the famous ADHM construction. So we stand before a natural question: How do the canonical initial condition and the canonical transition function for the ADHM solutions look like? The corresponding formula in the simplest one-instanton example was derived by Crane in [12], though in this paper the transition function is not recognized as canonical. Here we present the general result. But knowing the canonical initial condition, we are even able to solve the initial value problem explicitly and in this way to derive the transcription of the ADHM construction into the $J, W$, $\hat{W}$-formalism of the inverse scattering method.

First, let us introduce some notation related to the ADHM construction. For a quadrupole

$$
\left(\alpha_{1}, \alpha_{2}, a, b\right) \in \mathbb{C}^{c, c} \times \mathbb{C}^{\prime \cdot \cdot} \times \mathbb{C}^{\prime \cdot /} \times \mathbb{C}^{c, \imath}
$$

and $\bar{\jmath}=\left(z_{j}\right) \in \mathbb{C}^{4}$ set

$$
\begin{aligned}
& R(\overline{\mathrm{z}})=\left(\begin{array}{c}
\alpha_{1} z_{1}+\alpha_{2}^{\dagger} z_{2}-z_{3} \\
\alpha_{2} z_{1}-\alpha_{1}^{\dagger} z_{2}+z_{4} \\
a z_{1}-b^{\dagger} z_{2}
\end{array}\right), \\
& \Delta(\overline{\bar{z}})=R(\overline{\mathrm{z}})^{\dagger} R(\overline{\mathrm{z}}) .
\end{aligned}
$$

The moduli space $M(i, c)$ of framed instanton bundles on $\mathbb{P}^{3}$ is the quotient of the set of matrices $\left(\alpha_{1}, \alpha_{2}, a, b\right)$ satisfying

(i) the rank of $R(\bar{z})$ is equal to $c$ for all $\bar{z} \neq 0$,

(ii) $R(\tau \bar{z})^{\dagger} R(\bar{z})=0$ for all $\bar{z}$, or equivalently,

by the action of $\mathbf{U}(c)$ :

$$
\Delta(\tau \bar{\jmath})=\Delta(\overline{\mathfrak{z}}) \text { for all } \overline{\mathfrak{z}},
$$

$$
R(\bar{z}) \mapsto \operatorname{diag}(\mathbf{g}, \mathbf{g}, \mathbf{1}) R(\overline{3}) \mathbf{g}^{-1}, \quad \mathbf{g} \in \mathbf{U}(c),
$$

(cf. [11]). Let $Z \in \mathbb{P}^{3}$ be a point with homogeneous coordinates $\bar{\jmath}=\left(z_{1}, \ldots, z_{4}\right)$. Then the fibre $F_{Z}$ of the instanton bundle in the ADHM construction is given by

$$
F_{Z}=\operatorname{Ker} R(\tau \bar{z})^{\dagger} / \operatorname{Ran} R(\bar{z}),
$$

(cf. [20]). The Hermitian product in $F_{Z}$ is given by

$$
([\jmath],[t])=s^{\dagger}\left(1-R(\bar{z}) \Delta(\bar{z})^{-1} R(\bar{z})^{\dagger}\right) t .
$$

The following lemma characterizes the jumping lines.

Lemma 5.1. Let $\mathscr{L}$ be a line in $\mathbb{P}^{3}$ and $Y_{0}, Z_{0} \in \mathscr{L}$ be two different points with homogeneous coordinates $\overline{\mathfrak{y}}_{0}, \bar{z}_{0}$, respectively. Then the restriction of the bundle $\mathbf{F} \mid \mathfrak{L}$ is holomorphically trivial if and only if the matrix $R\left(\tau \overline{\mathfrak{y}}_{0}\right)^{\dagger} R\left(\overline{\mathfrak{z}}_{0}\right)$ is invertible.

Proof. $(\Rightarrow)$ Fix a basis in the fibre over $Z_{0}$ via the choice of a $(2 c+\imath) \times \imath$ matrix $N$ satisfying

$$
R\left(\tau \bar{z}_{0}\right)^{\dagger} N=0, \quad \operatorname{rank}\left(N, R\left(\bar{z}_{0}\right)\right)=\imath+c .
$$

Then the matrix-valued function

$$
S(Y)=\left[1-R\left(\overline{\mathrm{z}}_{0}\right)\left(R\left(\tau \overline{\mathfrak{y}}_{0}\right)^{\dagger} R\left(\overline{\mathrm{z}}_{0}\right)\right)^{-1} R\left(\tau \overline{\mathfrak{y}}_{0}\right)^{\dagger}\right] N
$$


modulo $\operatorname{Ran} R(\overline{\mathfrak{y}}), Y=\operatorname{span} \overline{\mathfrak{y}} \in \mathscr{L}$, is in fact a holomorphic trivialization of $F$ on $\mathscr{L}$.

$(\Leftrightarrow)$ Suppose $R\left(\tau \overline{\mathfrak{y}}_{0}\right)^{\dagger} R\left(\overline{\mathrm{z}}_{0}\right)$ is singular. We shall show that $F \mid \mathscr{L}$ has a nontrivial section with at least one zero. Put

$$
K=\operatorname{Ker} R\left(\tau \overline{\mathfrak{y}}_{0}\right)^{\dagger} R\left(\overline{\mathrm{z}}_{0}\right) \subset \mathbb{C}^{c} .
$$

Let $\Lambda$ be a projector onto $R\left(\bar{z}_{0}\right) K \subset \mathbb{C}^{2 c+\imath}$ according to any direct summand. Since the restriction $R\left(\bar{z}_{0}\right): K \rightarrow R\left(\bar{z}_{0}\right) K$ is an isomorphism, we can define the linear operator

$$
A=R\left(\overline{\mathrm{z}}_{0}\right)^{-1} \circ \Lambda \circ R\left(\overline{\mathfrak{y}}_{0}\right): K \rightarrow K .
$$

Choose an eigenvalue- $\kappa$ of the endomorphism $A$ and a vector $f \in K \backslash \operatorname{Ran}(A+\kappa \mathbf{1})$. Hence

The section

$$
R\left(\overline{\mathrm{z}}_{0}\right) f \neq\left(R\left(\overline{\mathfrak{y}}_{0}\right)+\kappa R\left(\overline{\mathrm{z}}_{0}\right)\right) g \text { for all } g \in K
$$

$$
\diamond(Y)=R\left(\bar{\jmath}_{0}\right) f \operatorname{modulo} \operatorname{Ran} R(\overline{\mathfrak{y}}), \quad Y=\operatorname{span} \overline{\mathfrak{y}} \in \mathscr{L},
$$

is holomorphic and $\varsigma\left(Z_{0}\right)=0$. Now it suffices to verify that $\varsigma(Y) \neq 0$, where $Y \in \mathscr{L}$ is the point with homogeneous coordinates $\overline{\mathfrak{y}}=\overline{\mathfrak{y}}_{0}+\kappa \overline{\mathfrak{z}}_{0}$.

Actually, provided there exists $g$ such that

we have

$$
R\left(\overline{\mathrm{z}}_{0}\right) f=R(\overline{\mathfrak{y}}) g=\left(R\left(\overline{\mathfrak{y}}_{0}\right)+\kappa R\left(\overline{\mathfrak{z}}_{0}\right)\right) g,
$$

$$
0=R\left(\tau \bar{z}_{0}\right)^{\dagger} R\left(\bar{z}_{0}\right) f=R\left(\tau \overline{\mathrm{z}}_{0}\right)^{\dagger} R\left(\overline{\mathfrak{y}}_{0}\right) g=-R\left(\tau\left(\overline{\mathfrak{y}}_{0}\right)^{\dagger} R\left(\overline{\mathrm{z}}_{0}\right) g .\right.
$$

Hence $g \in K$ and we get a contradiction.

Now it is enough to follow the construction of Chap. 4. Set

$$
R_{0}=\left(\begin{array}{l}
\mathbf{0} \\
1 \\
\mathbf{0}
\end{array}\right), \quad R_{\infty}=\left(\begin{array}{l}
1 \\
\mathbf{0} \\
\mathbf{0}
\end{array}\right), \quad N_{0}=\left(\begin{array}{l}
\mathbf{0} \\
\mathbf{0} \\
\mathbf{1}
\end{array}\right)
$$

where the dimensions of these matrices are

$$
(2 c+\imath) \times c, \quad(2 c+\imath) \times c \quad \text { and } \quad(2 c+\imath) \times \imath,
$$

respectively. The matrices $R_{0}=R(0,0,0,1), R_{\infty}=(0,0,-1,0)$ are related to the points $P_{0}, P_{\infty}$, respectively, and the columns of $N_{0}$ determine the distinguished frame on $\mathscr{L}_{0}$. Note that it holds

$$
R_{0}^{\dagger} R(\overline{\bar{z}})=R(\tau \bar{\jmath})^{\dagger} R_{\infty}, \quad \text { and } \quad N_{0} N_{0}^{\dagger}=1-R_{0} R_{0}^{\dagger}-R_{\infty} R_{\infty}^{\dagger} .
$$

The frames $\left.\left\{\jmath_{1}, \ldots,\right\lrcorner_{\imath}\right\}$ and $\left\{\hat{\sigma}_{1}, \ldots, \hat{\sigma}_{\imath}\right\}$ are determined by the matrix-valued functions $S(Z), \hat{S}(Z)$ modulo $\operatorname{Ran} R(\overline{\mathfrak{z}})$, respectively, where $(Z=\operatorname{span} \overline{\mathfrak{z}})$

$$
\begin{gathered}
S(Z)=\left[1-R_{\infty}\left(R(\tau \bar{z})^{\dagger} R_{\infty}\right)^{-1} R(\tau \bar{\jmath})^{\dagger}\right] N_{0}, \\
\hat{S}(Z)=\left[1-R_{0}\left(R(\tau \bar{\jmath})^{\dagger} R_{0}\right)^{-1} R(\tau \bar{\jmath})^{\dagger}\right] N_{0} .
\end{gathered}
$$

Actually, $S(Z)$ is well defined and holomorphic on $\mathscr{U}_{0}=\mathbb{P}^{3} \backslash \operatorname{Sing}\left(P_{\infty}\right)$ and $S(Z)$ $=N_{0}$ identically on the punctured line $\mathscr{L}_{0} \backslash\left\{P_{\infty}\right\}$. The relations $(5.3)$ imply (as it should be)

$$
\widehat{S}(\tau Z)^{\dagger} S(Z)=1 \text {, }
$$

and hence $S(Z)$ is regular on $\mathscr{U}_{0}$. Besides, $R(\tau \bar{z})^{\dagger} S(Z)=0$. An analogous discussion can be done for $\hat{S}(Z)$. 
According to the construction, the canonical transition function is given by

$$
\begin{gathered}
G_{c}(Z)=S(\tau Z)^{\dagger} S(Z), \\
G_{c}(Z)^{-1}=\hat{S}(\tau Z)^{\dagger} \hat{S}(Z) .
\end{gathered}
$$

Now it is enough to substitute (5.4) into (5.7):

$$
G_{c}(Z)=1-N_{0}^{\dagger} R(\bar{z})\left(R(\tau \bar{z})^{\dagger} R_{0}\right)^{-1}\left(R_{0}^{\dagger} R(\bar{\jmath})\right)^{-1} R(\tau \bar{z})^{\dagger} N_{0} .
$$

Taking (3.20) into account, we get the canonical initial condition

$$
\begin{aligned}
W_{c}^{(0)}(y, z, \lambda)= & 1+\lambda\left(a y-b^{\dagger} z\right)\left(1-\alpha_{1} y-\alpha_{2}^{\dagger} z\right)^{-1} \\
& \times\left(1+\lambda\left(\alpha_{2} y-\alpha_{1}^{\dagger} z\right)\right)^{-1}\left(b y+a^{\dagger} z\right), \\
W_{c}^{(0)}(y, z, \lambda)^{-1}= & 1-\lambda\left(a y-b^{\dagger} z\right)\left(1+\lambda\left(\alpha_{2} y-\alpha_{1}^{\dagger} z\right)\right)^{-1} \\
& \times\left(1-\alpha_{1} y-\alpha_{2}^{\dagger} z\right)^{-1}\left(b y+a^{\dagger} z\right) .
\end{aligned}
$$

According to (3.20),

$$
G_{c}(Z)=G_{c}(\xi, \eta, \zeta)=W_{c}^{(0)}\left(z_{1} / z_{3}, z_{2} / z_{3}, z_{3} / z_{4}\right),
$$

and we can rewrite (5.9)

$$
\begin{aligned}
G_{c}(Z)= & 1+\left(a z_{1}-b^{\dagger} z_{2}\right)\left(z_{3}-\alpha_{1} z_{1}-\alpha_{2}^{\dagger} z_{2}\right)^{-1} \\
& \times\left(z_{4}+\alpha_{2} z_{1}-\alpha_{1}^{\dagger} z_{2}\right)^{-1}\left(b z_{1}+a^{\dagger} z_{2}\right) .
\end{aligned}
$$

Using the Cramer rule, we find that the canonical transition function $G_{c}$ for ADHM instantons has the form (4.3), where

$$
\begin{aligned}
& p_{0}(\overline{\mathrm{z}})=\operatorname{det}\left(z_{3}-\alpha_{1} z_{1}-\alpha_{2}^{\dagger} z_{2}\right), \\
& p_{\infty}(\overline{\bar{z}})=\operatorname{det}\left(z_{4}+\alpha_{2} z_{1}-\alpha_{1}^{\dagger} z_{2}\right) .
\end{aligned}
$$

These expressions agree with the conjecture following the formula (4.3).

To find the solution with the initial condition (5.10) in the $J, W, \hat{W}$-formalism of the inverse scattering method one has to solve the Riemann-Hilbert problem, i.e., to find the Birkhoff decomposition (2.13):

$$
G_{c}(x, \lambda)=\hat{W}(x, \lambda)^{-1} J(x)^{-1} W(x, \lambda) .
$$

But in the general case, the Riemann-Hilbert problem is highly nontriviail. Fortunately, we can avoid it using the following

Lemma 5.2. Let $F$ be a local instanton bundle defined on a neighborhood of the line $\mathscr{L}_{0} \subset \mathbb{P}^{3}$ and let $\left\{\sigma_{1}, \ldots, \delta_{2}\right\}$ be a holomorphic frame in a neighborhood of the point $P_{0}$. Then the Gram matrix $H=\left(\varsigma_{j}, \diamond_{k}\right)$ has the form (in the coordinates $(x, \lambda)$, we now distinguish between $\lambda$ and $\bar{\lambda}$ )

$$
H(x, \lambda, \bar{\lambda})=W(x, \lambda)^{\dagger} J(x)^{-1} W(x, \lambda) .
$$

where $J(x)=J(x)^{\dagger}, W(x, 0)=1$ and $J, W$ solve (2.6). Hence

$$
J(x)^{-1}=H(x, 0,0), \quad J(x)^{-1} W(x, \lambda)=H(x, \lambda, 0) .
$$

Proof. The assertion follows immediately from the form of the Birkhoff decomposition (2.13) of the transition function and from the form of the gauge transformation of the Gram matrix:

$$
H(x, \lambda, \bar{\lambda}) \mapsto \Gamma(x, \lambda)^{\dagger} H(x, \lambda, \bar{\lambda}) \Gamma(x, \lambda) .
$$


Now we apply this lemma to the frame $S(Z)$ and $\hat{S}(\tau Z)^{\dagger}$. In the coordinates $(x, \lambda)$ we have $\overline{\bar{z}}=(\lambda y-\bar{z}, \lambda z+\bar{y}, \lambda, 1)$, and so

$$
\begin{aligned}
& J(x)^{-1}=\left.S(\overline{\bar{z}})^{\dagger}\left(\mathbf{1}-R(\overline{\bar{z}}) \Delta(\overline{\bar{z}})^{-1} R(\overline{\bar{z}})^{\dagger}\right) S(\overline{\bar{z}})\right|_{\lambda=\bar{\lambda}=0}, \\
& J(x)^{-1} W(x, \lambda)=\left.S(\overline{\bar{z}})^{\dagger}\left(\mathbf{1}-R(\overline{\mathrm{z}}) \Delta(\overline{\mathrm{z}})^{-1} R(\overline{\bar{z}})^{\dagger}\right) S(\overline{\bar{z}})\right|_{\bar{\lambda}=0}, \\
& J(x)=\left.\hat{S}(\tau \bar{z})^{\dagger}\left(\mathbf{1}-R(\tau \bar{\jmath}) \Delta(\bar{z})^{-1} R(\tau \bar{\jmath})^{\dagger}\right) \hat{S}(\tau \bar{z})\right|_{\lambda=\bar{\lambda}=0}, \\
& W(x, \lambda)^{-1} J(x)=\left.\widehat{S}(\tau \bar{z})^{\dagger}\left(\mathbf{1}-R(\tau \bar{\jmath}) \Delta(\bar{z})^{-1} R(\tau \bar{z})^{\dagger}\right) \hat{S}(\tau \bar{z})\right|_{\bar{\lambda}=0} .
\end{aligned}
$$

To get the explicit formulae we set

and hereinafter

$$
\begin{array}{cl}
\Psi=a y-b^{\dagger} z, & \Psi_{\sigma}=b y+a^{\dagger} z, \\
\Phi=\alpha_{2} y-\alpha_{1}^{\dagger} z, & \Phi_{\sigma}=\alpha_{1} y+\alpha_{2}^{\dagger} z,
\end{array}
$$

$$
\begin{aligned}
\Delta & =\left(1-\Phi_{\sigma}^{\dagger}\right)\left(1-\Phi_{\sigma}\right)+\Phi^{\dagger} \Phi+\Psi^{\dagger} \Psi \\
& =\left(1-\Phi_{\sigma}\right)\left(1-\Phi_{\sigma}^{\dagger}\right)+\Phi \Phi^{\dagger}+\Psi_{\sigma} \Psi_{\sigma}^{\dagger} .
\end{aligned}
$$

In this notation, (5.2) is equivalent to

and (5.10) can be rewritten

$$
\Psi_{\sigma} \Psi=\left[\Phi, \Phi_{\sigma}\right]
$$

$$
W_{c}^{(0)}(y, z, \lambda)=1+\lambda \Psi\left(1-\Phi_{\sigma}\right)^{-1}(1+\lambda \Phi)^{-1} \Psi_{\sigma} .
$$

After some straightforward computations we arrive at

Theorem 5.3. The following formulae together with (5.14), (5.15) provide the complete transcription of the ADHM construction into the $J, W$-formalism of the inverse scattering method:

$$
\begin{aligned}
& W(x, \lambda)=\mathbf{1}+\lambda\left(\Psi+\Psi_{\sigma}^{\dagger}\left(\mathbf{1}-\Phi_{\sigma}^{\dagger}\right)^{-1} \Phi\right) \Delta^{-1} \\
& \times\left(1+\lambda \Phi\left(1-\Phi_{\sigma}^{\dagger}\right)^{-1}\right)^{-1}\left(\Psi_{\sigma}-\Phi\left(1-\Phi_{\sigma}^{\dagger}\right)^{-1} \Psi^{\dagger}\right), \\
& W(x, \lambda)^{-1}=1-\lambda\left(\Psi+\Psi_{\sigma}^{\dagger}\left(1-\Phi_{\sigma}^{\dagger}\right)^{-1} \Phi\right) \\
& \times\left(1+\lambda\left(1-\Phi_{\sigma}^{\dagger}\right)^{-1} \Phi\right)^{-1} \Delta^{-1}\left(\Psi_{\sigma}-\Phi\left(1-\Phi_{\sigma}^{\dagger}\right)^{-1} \Psi^{\dagger}\right), \\
& J(x)=1+\Psi_{\sigma}^{\dagger}\left(1-\Phi_{\sigma}^{\dagger}\right)^{-1}\left(1-\Phi_{\sigma}\right)^{-1} \Psi_{\sigma} \\
& -\left(\Psi+\Psi_{\sigma}^{\dagger}\left(1-\Phi_{\sigma}^{\dagger}\right)^{-1} \Phi\right) \Delta^{-1}\left(\Psi^{\dagger}+\Phi^{\dagger}\left(1-\Phi_{\sigma}\right)^{-1} \Psi_{\sigma}\right), \\
& J(x)^{-1}=1+\Psi\left(1-\Phi_{\sigma}\right)^{-1}\left(1-\Phi_{\sigma}^{\dagger}\right)^{-1} \Psi^{\dagger} \\
& -\left(\Psi_{\sigma}^{\dagger}-\Psi\left(1-\Phi_{\sigma}\right)^{-1} \Phi^{\dagger}\right) \Delta^{-1}\left(\Psi_{\sigma}-\Phi\left(1-\Phi_{\sigma}^{\dagger}\right)^{-1} \Psi^{\dagger}\right) .
\end{aligned}
$$

If we put $\hat{W}(x, \lambda)=\left(W(x,-1 / \bar{\lambda})^{\dagger}\right)^{-1}$, then $(J, W, \hat{W}) \in \bar{\Xi}_{c}$ is the distinguished canonical solution defined in Chap 3.

Let us conclude this chapter with some examples. The most familiar self-dual solutions are 't Hooft's instantons with the gauge group $\mathbf{S U}(2)$. In this case

$$
\begin{gathered}
a=\left(\begin{array}{l}
\varrho \\
\mathbf{0}
\end{array}\right), \quad \ell=\left(\mathbf{0},-\varrho^{t}\right), \quad \varrho=\left(\varrho_{1}, \ldots, \varrho_{c}\right), \\
-\alpha_{1}=\operatorname{diag}\left(\bar{u}_{1}, \ldots, \bar{u}_{c}\right), \quad-\alpha_{2}=\operatorname{diag}\left(v_{1}, \ldots, v_{c}\right),
\end{gathered}
$$


where the constants $\varrho_{j}$ are positive and the points $\left(u_{j}, v_{j}\right) \in \mathbb{C}^{2}, 1 \leqq j \leqq c$, are mutually different. The canonical initial condition and the canonical transition function take the form

$$
\begin{gathered}
W_{c}^{(0)}(y, z, \lambda)=1+\lambda \sum_{j=1}^{c} \frac{\varrho_{j}^{2}}{\left(1+\bar{u}_{j} y+\bar{v}_{j} z\right)\left(1-\lambda\left(v_{j} y-u_{j} z\right)\right)}\left(\begin{array}{c}
y z-y^{2} \\
z^{2}-y^{z}
\end{array}\right), \\
G_{c}(\xi, \eta, \zeta)=1+\sum_{j=1}^{c} \frac{\varrho_{j}^{2}}{\left(\zeta+\bar{u}_{j} \xi+\bar{v}_{j} \eta\right)\left(1-v_{j} \xi+u_{j} \eta\right)}\left(\begin{array}{c}
\xi \eta-\xi^{2} \\
\eta^{2}-\xi \eta
\end{array}\right) .
\end{gathered}
$$

The modified 't Hooft's solutions (cf. [4]) are known to exhaust all twoinstantons (cf. [21]). Let $\left(u_{j}, v_{j}\right) \in \mathbb{C}^{2}, 0 \leqq j \leqq c$, be mutually different points and $\chi_{j}$, $0 \leqq j \leqq c$, be real parameters such that $\chi_{0}+\ldots+\chi_{c}=1,0<\chi_{j}<1$. Let

$$
V_{j}=\operatorname{span}\left\{u_{j} e_{1}+v_{j} e_{2}+e_{3},-\bar{v}_{j} e_{1}+\bar{u}_{j} e_{2}+e_{4}\right\}, \quad 0 \leqq j \leqq c,
$$

be the corresponding two-dimensional $\tau$-invariant subspace in $\mathbb{C}^{4}$. We furnish it with the Hermitian product

$$
\frac{\chi_{j}}{1+\left|u_{j}\right|^{2}+\left|v_{j}\right|^{2}}\langle\circ, 0\rangle,
$$

where $\langle\circ, 0\rangle$ is the restriction of the standard Hermitian product in $\mathbb{C}^{4}$, and let $(\circ, \circ)_{V}$ designate the induced Hermitian product in the direct sum $V=V_{0} \oplus \ldots \oplus V_{c}$. Then the instanton bundle is the quotient

$$
F=\tilde{F} / K,
$$

where for $Z=\operatorname{span} \bar{\jmath}, \bar{\jmath} \in \mathbb{C}^{4}$, the fibre $\widetilde{F}_{Z}$ is the $(c+2)$-dimensional subspace in $V$ determined by the equations

$$
\left(u_{0}, \ldots, u_{c}\right) \in \tilde{F}_{Z} \subset V \quad \text { iff }\left\langle\tau \bar{z}, u_{0}\right\rangle=\left\langle\tau \bar{z}, u_{1}\right\rangle=\ldots=\left\langle\tau \bar{z}, u_{c}\right\rangle,
$$

and $K_{Z}=\operatorname{span}\left(\psi_{1}, \ldots, \psi_{c}\right)$, where the vectors $\psi_{j} \in V, 1 \leqq j \leqq c$, are linearly independent and unambiguously determined by the equations

$$
\left(\psi_{j}, \varphi\right)_{V}=\left\langle\bar{z}, u_{j}-u_{0}\right\rangle \text { for all } \varphi=\left(u_{0}, \ldots, u_{c}\right) \in V .
$$

The Hermitian product in $F_{Z}$ is given by

$$
([\varphi],[\psi])=\left(\varphi,\left(1-\Lambda_{Z}\right) \psi\right),
$$

where $\Lambda_{Z}$ is the orthogonal projector onto the subspace in $V$ spanned by the vectors $\psi_{1}, \tau \psi_{1}, \ldots, \psi_{c}, \tau \psi_{c}$.

Now, the same procedure as before should follow but there is no need to give all the details. We only note that the following trivialization $\left.\left\{\varsigma_{1},\right\lrcorner_{2}\right\}$ appears to be convenient in the computations

$$
\begin{aligned}
s_{1}= & \left(\frac{1}{1-v_{0} \xi+u_{0} \eta}, 0, \ldots, \frac{1}{1-v_{c} \xi+u_{c} \eta}, 0\right), \\
s_{2}= & \left(\frac{\zeta+\bar{u}_{0} \xi+\bar{v}_{0} \eta}{1-v_{0} \xi+u_{0} \eta}, 1, \ldots, \frac{\zeta+\bar{u}_{c} \xi+v_{c} \eta}{1-v c \xi+u_{c} \eta}, 1\right) .
\end{aligned}
$$


The entries here are the coordinates with respect to the bases (5.20) in the vector spaces $V_{0}, \ldots, V_{c}$. After some algebra we get

$$
\begin{aligned}
W_{c}^{(0)}(y, z, \lambda)= & +\frac{\lambda \Theta}{\omega \omega_{\sigma}} \sum_{j<k} \frac{\chi_{j} \chi_{k}}{\vartheta_{j} \vartheta_{k}} \\
& \times\left(\begin{array}{cc}
-\left(\phi_{k}-\phi_{j}\right)\left(\phi_{\sigma, k}-\phi_{\sigma, j}\right) & -\left(\phi_{\sigma, k}-\phi_{\sigma, j}\right)^{2} \\
\left(\phi_{k}-\phi_{j}\right)^{2} & \left(\phi_{k}-\phi_{j}\right)\left(\phi_{\sigma, k}-\phi_{\sigma, j}\right)
\end{array}\right) \\
& -\frac{\lambda^{2} \Theta}{\omega \omega_{\sigma}} \sum_{i<j<k} \frac{\chi_{i} \chi_{j} \chi_{k}}{\vartheta_{i} \vartheta_{j} \vartheta_{k}}\left(\phi_{\sigma, i}\left(\phi_{j}-\phi_{k}\right)+\operatorname{cyk} 1(i j k)\right)^{2} \\
& \times\left(\begin{array}{ll}
0 & 0 \\
0 & 1
\end{array}\right),
\end{aligned}
$$

where we have put

$$
\begin{gathered}
\phi_{j}=v_{j} y-u_{j} z, \quad \phi_{\sigma, j}=\bar{u}_{j} y+\bar{v}_{j} z, \\
\vartheta_{j}=\left(1+\phi_{\sigma, j}\right)\left(1-\lambda \phi_{j}\right), \quad \Theta=\vartheta_{0} \ldots \vartheta_{c}, \\
\omega=\chi_{0}\left(1+\phi_{\sigma, 1}\right) \ldots\left(1+\phi_{\sigma, c}\right)+\operatorname{cykl}(01 \ldots c), \\
\omega_{\sigma}=\chi_{0}\left(1-\lambda \phi_{1}\right) \ldots\left(1-\lambda \phi_{c}\right)+\operatorname{cykl}(01 \ldots c) .
\end{gathered}
$$

\section{Conjecture: The Moduli Spaces as Algebraic Sets}

Owing to the Donaldson's result, we can consider the moduli space $\mathcal{O} M(\iota, c)$ of framed holomorphic vector bundles on $\mathbb{P}^{2}$ instead of the moduli space $M(\imath, c)$. The description of $\mathcal{O} M(\imath, c)$ is similar to that of $M(\imath, c)$ (cf. [11]). Theorem 4.4 asserts that the points from $\mathcal{O M}(r, c)$ are in one-to-one correspondence with the restricted canonical initial conditions $W_{\text {res }}^{(0)}:=\left.W_{c}^{(0)}\right|_{x=0}$. We introduce a new coordinate $w=\lambda y$ and define

From (5.10) it follows

$$
W_{\mathrm{res}}^{(0)}(y, w)=W_{c}^{(0)}(y, 0, w / y)
$$

$$
W_{\text {res }}^{(0)}(y, w)=1+\text { ywa }\left(1-\alpha_{1} y\right)^{-1}\left(1+\alpha_{2} w\right)^{-1} b .
$$

The Taylor expansion at the origin shows that a point from $\mathcal{O} M(\imath, c)$ corresponding to the equivalence class $\left[\left(\alpha_{1}, \alpha_{2}, a, \ell\right)\right]$ is unambiguously determined by the infinite doubly indexed sequence of matrices $a \alpha_{1}^{j} \alpha_{2}^{k} b, j, k=0,1, \ldots$ But this sequence can be replaced by a finite one.

Lemma 6.1. Let $f(t) \in \mathcal{O}_{0, \bar{\xi}}$ be a rational function in one variable over any field $\overline{\mathfrak{f}}$ of characteristic zero and assume $f(t)$ to be regular at the origin. If $f$ can be expressed as a quotient $f=p / q$ with $p, q$ being polynomials with degrees less than or equal to $n$ then $f$ is unambiguously determined by $2 n$ numbers $f_{j}=\frac{1}{j !} \frac{d^{j}}{d t^{j}} f(0), 0 \leqq j \leqq 2 n-1$.

Proof. Put

$$
M(k)=\left(\begin{array}{ccc}
f_{k} & \ldots & f_{1} \\
f_{k+1} & \ldots & f_{2} \\
\ldots & \ldots & \ldots \\
f_{2 k-1} & \ldots & f_{k}
\end{array}\right)
$$


and for two polynomials

$$
p(t)=p_{0}+p_{1} t+\ldots+p_{k} t^{k}, \quad q(t)=1+q_{1} t+\ldots+q_{k} t^{k},
$$

denote

$$
\mathbf{R}(p, q)=\left(\begin{array}{ll}
A & C \\
B & D
\end{array}\right)
$$

where the blocks $A, B$ are defined

$$
A=\left(\begin{array}{cccc}
p_{0} & & & 0 \\
p_{1} & p_{0} & & \\
\vdots & \vdots & & \\
p_{k-1} & p_{k-2} & \ldots & p_{0}
\end{array}\right), \quad B=\left(\begin{array}{cccc}
p_{k} & p_{k-1} & \ldots & p_{1} \\
& p_{k} & \ldots & p_{2} \\
& & & \vdots \\
0 & & & p_{k}
\end{array}\right),
$$

and the blocks $C, D$ are defined analogously but now with the polynomial $q$. Then $\operatorname{det} \mathbf{R}(p, q)$ as a polynomial in the coefficients $p_{j}, q_{j}$ is the resultant of the polynomials $p, q$ and it vanishes if and only if $p_{k}=q_{k}=0$ or $p, q$ have a common nontrivial divisor (cf. [22], Sect. 34). It holds

If $f=p / q$, then $\operatorname{det} \mathbf{R}(p, q)=(-1)^{k} \operatorname{det} M(k)$.

Actually, the recurrence relations

$$
\begin{gathered}
0=p_{0}-f_{0}, \\
0=p_{j}-f_{j}-\sum_{i=0}^{j-1} f_{i} q_{j-1}, \quad j \geqq 1\left(p_{i}=q_{i}=0 \text { for } i>k\right),
\end{gathered}
$$

imply

$$
\begin{aligned}
\operatorname{det} \mathbf{R}(p, q) & =\operatorname{det}\left(\begin{array}{lll}
A & C & \mathbf{0} \\
B & D & C \\
\mathbf{0} & \mathbf{0} & \mathbf{1}
\end{array}\right)=\operatorname{det}\left(\begin{array}{ccc}
\mathbf{0} & C & \mathbf{0} \\
\mathbf{0} & D & C \\
-M(k) & \mathbf{0} & \mathbf{1}
\end{array}\right) \\
& =(-1)^{k} \operatorname{det} M(k) .
\end{aligned}
$$

Write now $f$ as the quotient $f=p / q$ with both the numerator and the denominator being uniquely characterized by the condition: $p, q$ have no common nontrivial divisor and $q(0)=1$. Put $m=\max (\operatorname{deg} p, \operatorname{deg} q)$. Then $m$ is the lowest non-negative integer with the property: $\operatorname{det} M(k)=0$ for all $k>m$. From (6.3) it follows that the coefficients $\left(p_{0}, \ldots, p_{m}, q_{1}, \ldots, q_{m}\right)$ solve the system of linear equations with the matrix

$$
\left(\begin{array}{cc}
1 & -E \\
\mathbf{0} & -M(m)
\end{array}\right) \text {, where } \quad \boldsymbol{E}=\left(\begin{array}{ccc}
0 & \\
f_{0} & \\
f_{1} & f_{0} \\
\ldots \ldots & \ldots \ldots \ldots \\
f_{m-1} & f_{m-2} \ldots & f_{0}
\end{array}\right) \text {, }
$$

and with the right-hand side $\left(f_{0}, \ldots, f_{2 m-1}\right)$. For $m=0$ the blocks $0,-E,-M(m)$ in (6.4) are empty, for $m>0$ the matrix (6.4) is regular. Hence the coefficients $p_{j}, q_{j}$ can be reconstructed in the unique way.

Now we can construct two holomorphic embeddings of the moduli space $\mathcal{O} M(\iota, c)$ into a finite-dimensional vector space. 
Proposition 6.2. The holomorphic mapping $\mathcal{O} M(\imath, c) \rightarrow \mathbb{C}^{4 c^{2} \imath^{2}}$ :

$$
\left[\left(\alpha_{1}, \alpha_{2}, a, b\right)\right] \mapsto\left(a \alpha_{1}^{j} \alpha_{2}^{k} b ; 0 \leqq j, k \leqq 2 c-1\right)
$$

is injective.

Proof. Use the Cramer rule and apply twice Lemma 6.1. First, putting $\overline{\mathfrak{f}}=\mathbb{C}$ we reconstruct $a\left(1-\alpha_{1} y\right)^{-1} \alpha_{2}^{k} b, 0 \leqq k \leqq 2 c-1$. Then putting $\overline{\mathfrak{f}}=\mathbb{C}(y)-$ the field of complex rational functions in the variable $y$ - we reconstruct $\alpha\left(1-\alpha_{1} y\right)^{-1}\left(1+\alpha_{2} w\right)^{-1} b$.

Let us now consider another and maybe more fruitful mapping. The Cramer rule implies

$$
\begin{aligned}
W_{\mathrm{res}}^{(0)}(y, w)= & +\left(1+\sum_{j=1}^{c} s_{j} y^{j}\right)^{-1}\left(1+\sum_{j=1}^{c} t_{j} w^{j}\right)^{-1} \\
& \times \sum_{j, k=1}^{c} \mathscr{R}_{j k} y^{j} w^{k}
\end{aligned}
$$

where $s_{j}, t_{j} \in \mathbb{C}, \mathscr{R}_{j k} \in \overline{\mathrm{g}} \overline{\mathrm{l}}(\iota, \mathbb{C})$ and

$$
1+\sum s_{j} y^{j}=\operatorname{det}\left(1-\alpha_{1} y\right), \quad 1+\sum t_{j} w^{j}=\operatorname{det}\left(1+\alpha_{2} w\right) .
$$

Proposition 6.3. The mapping $\mathcal{O} M(\imath, c) \rightarrow \mathbb{C}^{2 c+\iota^{2} c^{2}}$ :

$$
\left[\left(\alpha_{1}, \alpha_{2}, a, b\right)\right] \mapsto\left(s_{j}, t_{j}, \mathscr{R}_{j k} ; 1 \leqq j, k \leqq c\right)
$$

is holomorphic and injective. Moreover, it is a homeomorphism onto its image. This image is an irreducible locally analytic set in $\mathbb{C}^{2 c+\iota^{2} c^{2}}$ and its dimension is equal to $\operatorname{dim} \mathcal{O} M(\imath, c)$.

Remark. Recall that the moduli spaces are known to be connected (cf. [16], Chap. II).

Proof. The interpretation of $W_{\text {res }}^{(0)}$ as a transition function shows that this mapping is a homeomorphism onto its image. All the properties of this image are then characterized by Remmert's Theorem (cf. [19], Sect. IV.7).

Up to the end of this chapter, we identify $\mathcal{O} M(r, c)$ with its image in the mapping (6.7). The canonical transition function on $\mathbb{P}^{2}$ corresponding to the initial condition $W_{\text {res }}^{(0)}$ has the form

where

$$
G_{\text {res }}(\overline{\bar{z}})=1+\frac{1}{\not_{0}(\overline{\mathrm{z}}) \not_{\infty}(\overline{\mathrm{z}})} \mathscr{R}(\overline{\mathrm{z}}), \quad \overline{\mathrm{z}}=\left(z_{1}, z_{2}, z_{3}, z_{4}\right),
$$

$$
\begin{gathered}
\not_{0}(\overline{\bar{z}})=z_{3}^{c}+\sum_{j=1}^{c} S_{j} z_{1}^{j} z_{3}^{c-j}, \\
\not_{\infty}(\overline{3})=z_{4}^{c}+\sum_{j=1}^{c} t_{j} z_{1}^{j} z_{4}^{c-j}, \\
\mathscr{R}(\overline{\bar{z}})=\sum_{j, k=1}^{c} \mathscr{R}_{j k} z_{1}^{j+k} z_{3}^{c-j} z_{4}^{c-k} .
\end{gathered}
$$

This is in agreement with the conjecture following the expression (4.3). This transition function defines a holomorphic vector bundle $F$ on the set $\mathbb{P}^{2} \backslash \mathscr{M}$, with $\mathscr{M}$ being the discrete set

$$
\mathscr{M}: p_{0}(\bar{\jmath})=p_{\infty}(\bar{\jmath})=0 \text {. }
$$


Now we state two problems:

(1) The first problem naturally arising is to determine for which parameters $\left(s_{j}, t_{j}, \mathscr{R}_{j k}\right)$ the singular points from the set $\mathscr{M}$ are removable in the sense that $l_{*} \mathscr{F}$ is a locally free rank- $r$ sheaf on $\mathbb{P}^{2}$. Here $\imath: \mathbb{P}^{2} \backslash \mathscr{M} \hookrightarrow \mathbb{P}^{2}$ is the embedding and $\mathscr{F}$ is the sheaf of germs of holomorphic sections in $F$. Owing to Hartog's Theorem on removability of singularities (cf. [19], Sect. IV.2), we can simplify this discussion. The singularity in the point $Q \in \mathscr{M}$ is removable if and only if the bundle $F$ is holomorphically trivial on some punctured neighborhood $\mathscr{U} \backslash\{Q\}$ of the point $Q$. (2) For such parameters it is further necessary to compute the second Chern number $c_{2}(F)$. Let $\mathbb{P}^{2} \subset \mathbb{P}^{2} \times \mathbb{P}^{1}$ be the blow-up of $\mathbb{P}^{2}$ at the point $z_{1}=z_{3}=0$. Here we can identify $\mathbb{P}^{1}$ with the line $\mathbb{P}\left(e_{1}, e_{3}\right) \subset \mathbb{P}^{2}$. Then $R_{1} \mathrm{pr}_{2 *} \mathrm{pr}_{1}^{*} \mathscr{F}(-1)$ is a sheaf on $\mathbb{P}\left(e_{1}, e_{3}\right)$ with the support being contained in the discrete set $p_{0}\left(z_{1}, z_{3}\right)=0$ and the sum of dimensions of the stalks over the points from this discrete set equals to $c_{2}(F)$ (this assertion is proven in [16], Chap. II).

Conjecture. The equality $c_{2}(F)=c$ should lead to a system of algebraic equations specifying $\mathcal{O} M(\iota, c)$ as an algebraic set.

We solve the problem (1) for the lowest rank $\iota=2$. Up to the end of this chapter, all matrices are supposed to belong to $\bar{g} \bar{l}(2, \mathbb{C})$ and the same is true for the values of matrix-valued functions. First, note that the equality $\operatorname{det} G_{\text {res }}=1$ (Proposition 4.3) leads to the equations

$$
\begin{gathered}
\operatorname{tr} \mathscr{R}_{11}=\operatorname{tr} \mathscr{R}_{1 j}=\operatorname{tr} \mathscr{R}_{j 1}=0, \quad 2 \leqq j \leqq c, \\
\left(1+\sum_{j=1}^{c} s_{j} y^{j}\right)\left(1+\sum_{j=1}^{c} t_{j} w^{j}\right) \sum_{j, k=2}^{c} \operatorname{tr} \mathscr{R}_{j k} \mathscr{Y}^{j-2} w^{k-2} \\
+\operatorname{det}\left(\sum_{j, k=1}^{c} \mathscr{R}_{j k} \mathscr{Y}^{j-1} w^{k-1}\right)=0 .
\end{gathered}
$$

Equation (6.11) means that all coefficients of the polynomial in the variables $y, w$ on the left-hand side are equal to zero. So Eqs. (6.10), (6.11) represent together $2 c(2 c-1)$ algebraic conditions on the $2 c(2 c+1)$ independent variables $\left(s_{j}, t_{j}, \mathscr{R}_{j k}\right)$. If we subtract these numbers we get exactly the dimension of the complex space $\mathcal{O} M(r, c)$ which is known to be $4 c$ [11]. This fact conjectures that the moduli space $\mathcal{O} M(r, c)$ might be identified with a set $\mathscr{A} \backslash \mathscr{B}$, where $\mathscr{A}$ is an irreducible component of the algebraic set (6.10), (6.11) and $\mathscr{B}$ is an algebraic set. The one- and twoinstanton examples which are mentioned in Chap. 8 support this conjecture.

Now we shall show that the condition $\operatorname{det} G_{\text {res }}=1$ is sufficient for the removability of the singular points. The problem is local. We choose coordinates $u, v$ on a neighborhood $\mathscr{U}$ of the singular point $Q \in \mathscr{M}$ in such a way that the algebraic set $h_{0}(\bar{\jmath})=0$ (respectively $\not_{\infty}(\bar{z})=0$ ) coincides on $\mathscr{U}$ with $u=0$ (respectively $v=0$ ) and hence $Q$ has the coordinates $u=v=0$. The vector bundle $F$ is holomorphically trivial on $\mathscr{U} \backslash\{Q\}$ if and only if the transition function $G_{\text {res }}$ has a special decomposition as the following proposition asserts.

Proposition 6.4. Let $G(u, v)$ be a $2 \times 2$ matrix-valued function defined on a neighborhood of the origin in $\mathbb{C}^{2}$, with the properties:

(i) $G$ is meromorphic with the poles on the set $u v=0$,

(ii) $\operatorname{det} G \equiv 1$.

Then there exists a decomposition $G=X Y$, where $X, Y$ are again unimodular meromorphic matrix-valued functions and $X$ (respectively $Y$ ) has the pole on the line $u=0$ (respectively $v=0$ ). 
Proof. The function $G(u, v)$ can be written in the form

$$
G(u, v)=v^{-k} A(u)+v^{-k+1} B(u)+\ldots, \quad A(u) \neq \mathbf{0}, \quad k \geqq 0 .
$$

We can suppose that $k>0$ (the case $k=0$ is trivial) and proceed by induction. So it is sufficient to prove the inductive step:

There exists a matrix-valued function $H(u, v)$ which is unimodular and meromorphic with the pole on the line $v=0$ and such that the product $G H$ has the poles again on the set $u v=0$ but the order of the pole on the line $v=0$ is equal at most to $(k-1)$.

$$
\begin{gathered}
\text { Denote } J=\left(\begin{array}{rr}
0 & 1 \\
-1 & 0
\end{array}\right) \text {. The unimodularity of } G \text { implies: } \\
\qquad \operatorname{det} A=0, \quad \operatorname{tr}\left(A J B^{t} J\right)=0 .
\end{gathered}
$$

From the former equality it follows that there exists a holomorphic unimodular matrix-valued function $T(u)$ such that the second column of $A(u) T(u)$ vanishes. So we can suppose that

$$
A(u)=\left(\begin{array}{ll}
a(u) & 0 \\
b(u) & 0
\end{array}\right),
$$

and, again without loss of generality, that $a(u) \neq 0$. Let us denote the entries of $B$

$$
B(u)=\left(\begin{array}{ll}
* & c(u) \\
* & d(u)
\end{array}\right)
$$

(the first column is unessential) and put $f(u)=-c(u) / a(u)$. Note that the latter equality in (6.10) means that $a d-b c=0$ and hence $a f+c=0, b f+d=0$. Now we distinguish two possibilities. First, provided $f(u)$ is holomorphic, we can put

$$
H(u, v)=\left(\begin{array}{cc}
f(u) & -v \\
v^{-1} & 0
\end{array}\right) .
$$

Second, provided $f(u)$ has a pole in the origin, i.e., $f(u)=u^{-j} g(u), g(0) \neq 0, j>0$, we can put

$$
H(u, v)=\left(\begin{array}{cc}
g(u) & 0 \\
u^{j} v^{-1} & g(u)^{-1}
\end{array}\right) .
$$

\section{On the Action of the Loop Group}

This chapter serves as an illustration of another possible application of the results from Chap. 3. Here we try to prolong the discussion initiated by Crane in [12] about the loop group action on instanton solutions.

Notation. The embedding of the space $\mathbb{\mathfrak { S }}$ into the vector space

$$
\overline{\mathfrak{g}} \overline{\mathfrak{I}}(\iota, \mathbb{C} \llbracket x \rrbracket) \times \overline{\mathfrak{g}} \overline{\mathrm{l}}(\iota, \mathbb{C} \llbracket x, \lambda \rrbracket) \times \overline{\mathfrak{g}} \overline{\mathfrak{l}}\left(\iota, \mathbb{C} \llbracket x, \lambda^{-1} \rrbracket\right)
$$

enables to treat formally the Lie algebra $\overline{\mathfrak{X}}(\overline{\mathfrak{S}})$ of vector fields on $\mathbb{\mathfrak { S }}$. Denote by $\overline{\mathfrak{g}} \overline{\mathrm{I}}\left(\imath, \mathbb{C}\left[\lambda, \lambda^{-1}\right]\right)$ the Lie algebra of Lorain polynomials in the variable $\lambda$ with coefficients from the Lie algebra $\overline{\mathfrak{g}} \mathrm{T}(\imath, \mathbb{C})$. Dolan [8] and Chau et al. [9] discovered the following infinitesimal action, i.e., a Lie algebra homomorphism

$$
\delta: \overline{\mathfrak{g}} \overline{\mathrm{T}}\left(\imath, \mathbb{C}\left[\lambda, \lambda^{-1}\right]\right) \rightarrow \overline{\mathfrak{X}}(\overline{\mathfrak{S}}): T \lambda^{-k} \mapsto\left(\delta_{k}(T)_{J}, \delta_{k}(T)_{W}, \delta_{k}(T)_{\hat{W}}\right),
$$


where $k \in \mathbb{Z}, T \in \overline{\mathfrak{g}}\left(\langle, \mathbb{C})\right.$. Introduce the $\overline{\mathfrak{g}} \mathfrak{\mathbb { l }}(\nu, \mathbb{C}[x])$-valued functions $d_{k}(T), \hat{d}_{k}(T)$, $k \in \mathbb{N}_{0}$, defined on $\overline{\mathcal{S}}$ and linearly depending on the parameter $T \in \overline{\mathfrak{g}}(\iota, \mathbb{C})$, by the relations:

$$
\begin{gathered}
W(\lambda) T W(\lambda)^{-1}=\sum_{k=0}^{\infty} d_{k}(T) \lambda^{k}, \\
\hat{W}(\lambda) T \hat{W}(\lambda)^{-1}=\sum_{k=0}^{\infty} \hat{d}_{k}(T)(-\lambda)^{-k},
\end{gathered}
$$

for $(J, W, \hat{W}) \in \mathbb{\Xi}$. To simplify notation, we stress explicitly the dependence only on the variable $\lambda$ and not on the variables $x$. The vector fields $\delta_{k}(T)$ have the form

$$
\begin{gathered}
\delta_{k}(T)_{J}=d_{k}(T) J, \quad \text { for } \quad k>0, \\
=[T, J], \quad k=0, \\
=(-1)^{k+1} J \hat{d}_{-k}(T), \quad k<0, \\
\delta_{k}(T)_{W}=-\sum_{j=1}^{\infty} d_{k+j}(T) \lambda^{j} W(\lambda), \quad \text { for } k \geqq 0, \\
=\lambda^{-k}\left\{-W(\lambda) T+J \sum_{j=0}^{-k+1} \hat{d}_{j}(T)(-\lambda)^{-1} J^{-1} W(\lambda)\right\}, \quad k<0, \\
\delta_{k}(T)_{\hat{W}}=\lambda^{-k}\left\{-\hat{W}(\lambda) T+J^{-1} \sum_{j=0}^{k-1} d_{j}(T) \lambda^{j} J \hat{W}(\lambda)\right\}, \quad \text { for } \quad k>0, \\
=(-1)^{k+1} \sum_{j=1}^{\infty} \hat{d}_{-k+j}(T)(-\lambda)^{-j} \hat{W}(\lambda), \quad k \leqq 0 .
\end{gathered}
$$

It was also recognized by Chau et al. that to get a well defined action on the subspace $\overline{\mathfrak{S}}_{u} \subset \overline{\mathbb{S}}$ one must restrict $\overline{\mathrm{g}}\left(\iota, \mathbb{C}\left[\lambda, \lambda^{-1}\right]\right)$ to the subalgebra consisting of those elements $\sum T_{k} \lambda^{-k}$ which satisfy

$$
T_{-k}=(-1)^{k+1} T_{k}^{\dagger} .
$$

Let $\Omega$ designate the loop group consisting of holomorphic mappings from $\mathbb{C}$ to $\mathbf{G L}(\iota, \mathbb{C})$ defined on a neighborhood of the unit circle.

Crane discovered that one is able to exponentiate the infinitesimal action if we replace $\overline{\mathfrak{S}}$ by its subspace of convergent power series $\overline{\mathfrak{G}}$. Let

$$
\begin{gathered}
G=(J \hat{W})^{-1} W \in \overline{\mathfrak{G}}, \quad T(\lambda)=\sum T_{k} \lambda^{-k} \in \overline{\mathfrak{g}} \overline{\mathfrak{l}}\left(\imath, \mathbb{C}\left[\lambda, \lambda^{-1}\right]\right), \\
\left(J^{\prime}, W^{\prime}, \hat{W}^{\prime}\right)=(J, W, \hat{W})+\varepsilon \delta(T(\lambda)), \quad \varepsilon \text {-infinitesimal } .
\end{gathered}
$$

Then using (7.3) we get after some algebra

$$
G^{\prime}:=\left(J^{\prime} \hat{W}^{\prime}\right)^{-1} W^{\prime}=(1+\varepsilon T(\lambda)) G(1-\varepsilon T(\lambda)) \quad\left(\bmod \varepsilon^{2}\right) .
$$

Hence the global action of $\Omega$ on $\overline{\mathfrak{5}}$ should be defined as:

$$
(g \cdot G)(x, \lambda)=g(\lambda) G(x, \lambda) g(\lambda)^{-1},
$$

where $g \in \Omega, G \in \overline{\mathfrak{G}}$. This definition is easily seen to be correct. Crane defined the action of $\Omega$ on $\widetilde{\mathfrak{E}}_{u}$ as follows:

$$
(g \cdot G)(x, \lambda)=g(\lambda) G(x, \lambda) g(-1 / \bar{\lambda})^{\dagger} .
$$

But the relation (7.6) suggests that this result requires an additional specification. 
The global action (7.6) will be correctly defined on the subspace $\overline{\mathfrak{G}}_{u} \subset \overline{\mathfrak{C}}$ if we restrict the group $\Omega$ to its subgroup $\Omega_{u}$ consisting of those elements $g(\lambda)$ which satisfy

$$
g(-1 / \bar{\lambda})^{\dagger}=g(\lambda)^{-1}
$$

This condition is the global counterpart to (7.4).

The main disadvantage is that the loop group action is gauge dependent and the moduli spaces being embedded into $\overrightarrow{\mathfrak{G}}_{u}$ are not invariant with respect to this action. Let us notice more closely the infinitesimal action on the one-instanton moduli space $M(2,1) \subset \mathbb{\Xi}_{c} \subset \overline{\mathfrak{S}}_{u}$. The action of $\Omega_{u}$ includes as a special case the adU(2)-transformations corresponding to the unitary transformations of the distinguished frame and there is no necessity to pursue them further. Because $d_{k}(\mathbf{1})$ $=\hat{d}_{k}(\mathbf{1})=0$ for $k>0$, it is enough to consider only vector fields $\delta(T(\lambda))$, where

Denote

$$
\begin{gathered}
T(\lambda)=\sum_{k \geqq 1}\left(T_{k} \lambda^{-k}+(-1)^{k+1} T_{k}^{\dagger} \lambda^{k}\right), \quad T_{k} \in \overline{\mathfrak{s}} \bar{l}(2, \mathbb{C}) . \\
\tilde{\delta}_{k}(T):=\delta_{k}\left(T \lambda^{-k}+(-1)^{k+1} T^{\dagger} \lambda^{k}\right), \\
\delta_{k}^{(0)}(T):=\operatorname{pr}_{c *} \tilde{\delta}_{k}(T), \quad k \geqq 1, \quad T \in \overline{\mathfrak{s}}(2, \mathbb{C}) .
\end{gathered}
$$

The projection $\mathrm{pr}_{c}: \overline{\mathfrak{S}}_{u} \rightarrow \overline{\mathfrak{S}}_{c}$ is defined in Chap. 3 (cf. (3.15)). Since $\mathbb{\mathfrak { S }}_{c} \cong \overline{\mathfrak{W}}$, we can regard the vector field $\delta_{k}^{(0)}(T)$ as being defined on $\overline{\mathfrak{W}}$.

We shall try to answer the following question: Which vector fields $\tilde{\delta}(T(\lambda)):=\sum_{k \geqq 1} \widetilde{\delta}_{k}\left(T_{k}\right)$ having been restricted to $M(2,1)$ are tangent to $\mathrm{pr}_{c}^{-1}(M(2,1))$ $\subset \mathbb{\mathfrak { S }}_{u}$, i.e., which vector fields $\delta^{(0)}(T(\lambda))$ are tangent to $M(2,1) \subset \overline{\mathfrak{B}}$ ? Roughly speaking, we ask when the loop group action preserves infinitesimally the topological charge finite and equal to one.

To this end we derive some necessary formulae. Let $(J, W, \hat{W}) \in \bar{\Xi}_{c}$ and $W_{c}^{(0)}$ be the corresponding canonical initial condition. Then it holds:

$$
\hat{d}_{k}\left(x ; T^{\dagger}\right)=d_{k}(x ; T)^{\dagger} \quad \text { and } \quad \hat{d}_{k}(y, z, 0,0 ; T)=0, \text { for } k \geqq 1 .
$$

Use these relations to get

$$
\begin{aligned}
\delta_{k}^{(0)}(T)= & -\sum_{j=1}^{\infty} d_{k+j}^{(0)}(y, z ; T) \lambda^{j} W^{(0)}(y, z, \lambda) \\
& -W^{(0)}(y, z, \lambda) \sum_{j=1}^{\infty} d_{k+j}^{(0)}(\bar{\lambda} \bar{z},-\bar{\lambda} \bar{y} ; T)^{\dagger}(-\lambda)^{j},
\end{aligned}
$$

where $d_{k}^{(0)}(y, z ; T):=d_{k}(y, z, 0,0 ; T)$. It is easy to show that

$$
d_{k}^{(0)}(y, z ; T) \in \overline{\mathfrak{s}} \bar{l}\left(2,(y, z)^{k+1}\right) .
$$

Here again $(y, z)$ is the ideal generated by the terms $y$, $z$. The infinitesimal canonical gauge transformation

$$
(J, W, \hat{W})+\varepsilon \widetilde{\delta}_{k}(T) \mapsto(J, W, \hat{W})+\varepsilon \delta_{k}^{(0)}(T), \quad \varepsilon \text {-infinitesimal },
$$

takes the form

$$
\Gamma_{-c}(\xi, \eta, \zeta)=\mathbf{1}+\varepsilon \sum_{j=0}^{k-1} d_{k-j}^{(0)}(\bar{\eta},-\bar{\xi} ; T)^{\dagger}(-\zeta)^{j} \quad\left(\bmod \varepsilon^{2}\right)
$$


The one-instanton canonical initial conditions depend on four complex parameters $e, f, u, v,(e, f) \neq 0$ :

where

$$
W_{c}^{(0)}(y, z, \lambda)=1+\frac{\lambda}{\left(1+\phi_{\sigma}\right)(1-\lambda \phi)} \Xi,
$$

$$
\begin{gathered}
\Xi=\left(\begin{array}{l}
e y-\bar{f} z \\
f z+\bar{e} z
\end{array}\right)(f y+\bar{e} z,-e y+\bar{f} z), \\
\phi=v y-u z, \quad \phi_{\sigma}=\bar{u} y+\bar{v} z .
\end{gathered}
$$

We obtain $(k \geqq 1)$

$$
\begin{gathered}
d_{k}^{(0)}(T)=\frac{\phi^{k-1}}{1+\phi_{\sigma}}[\Xi, T]-(k-1) \frac{\phi^{k-2}}{\left(1+\phi_{\sigma}\right)^{2}} \Xi T \Xi \\
\delta_{k}^{(0)}(T)=-\frac{\lambda \phi^{k-1}}{\left(1+\phi_{\sigma}\right)^{2}(1-\lambda \phi)}\left\{\phi\left(1+\phi_{\sigma}\right)[\Xi, T]-k \Xi T \Xi\right\} \\
+\frac{\lambda^{k+1} \phi_{\sigma}^{k-1}}{\left(1+\phi_{\sigma}\right)(1-\lambda \phi)^{2}}\left\{\phi_{\sigma}(1-\lambda \phi)\left[\Xi, T^{\dagger}\right]-\lambda k \Xi T^{\dagger} \Xi\right\} .
\end{gathered}
$$

Since $\delta_{k}^{(0)}(T) \in \overline{\bar{s}} \bar{T}\left(2,(y, z)^{k+2}\right)$, the infinite series $\sum_{k=1}^{\infty} \delta_{k}^{(0)}\left(T_{k}\right)=\delta^{(0)}(T(\lambda))$ makes sense in the realm of formal power series. The four-dimensional tangent space at the point $W_{c}^{(0)} \in M(2,1) \subset \overline{\mathfrak{B}}$ can be obtained by variation of the parameters $e, f, u, v$.

Comparing homogeneous terms of the forma power series, after rather straightforward considerations we arrive at the following conclusion.

Provided $T(\lambda)$ is nonzero, the vector field $\delta^{(0)}(T(\lambda))$ is tangent to the manifold $M(2,1)$ only in the points corresponding to the parameters $u=v=0$, e, f-arbitrary, and only in the case $T_{1}=0$. The vector fields $\delta_{k}^{(0)}(T), k \geqq 2$, vanish in these points and hence the vector fields $\widetilde{\delta}_{k}(T), k \geqq 2$, are tangent to the fibres of the projection $\mathrm{pr}_{c}$. The corresponding infinitesimal canonical transformation is

$$
\Gamma_{c}(\xi, \eta, \zeta)=\mathbf{1}-\varepsilon\left\{\left[Y, T^{\dagger}\right] \zeta+Y T^{\dagger} Y\right\}(-\zeta)^{k-2} \quad\left(\bmod \varepsilon^{2}\right),
$$

where $Y=\left.\Xi\right|_{y=\xi, x=\eta}$.

\section{Concluding Remark}

The basic notions this paper was dealing with are the canonical initial condition and the canonical transition function. And perhaps the most interesting conclusion we can derive from this discussion is to stress once more the conjecture we have proposed in Chap. 6. Namely, the following one- and two-instanton examples aim to support the idea that it should be possible to describe the moduli spaces $\mathcal{O} M(\imath, c)$ directly as algebraic sets. The one-instanton example is rather trivial. In the case of the two-instanton example, we present only the final formulae without other details but with the hope that in a forthcoming paper the complete results will appear.

Let $r=2$ and $c=1$. Then $\mathcal{O} M(2,1)$ being embedded into the vector space $\mathbb{C}^{6}$ with the coordinates $s_{1}, t_{1} \in \mathbb{C}, \mathscr{R}_{11} \in \mathbb{C}^{2,2}$, is biholomorphically equivalent to the 
set $\mathscr{A} \backslash \mathscr{B}$, where $\mathscr{A}$ is the four-dimensional algebraic set determined by the equation

$$
\operatorname{tr} \mathscr{R}_{11}=\operatorname{det} \mathscr{R}_{11}=0 \text {, or equivalently, } \mathscr{R}_{11}^{2}=0,
$$

and the algebraic set $\mathscr{B}$ is determined by the equation $\mathscr{R}_{11}=0$.

Let $\iota=2$ and $c=2$. Then $\mathcal{O} M(2,2)$ being embedded into the vector space $\mathbb{C}^{20}$ with the coordinates $s_{1}, t_{1}, s_{2}, t_{2} \in \mathbb{C}, \mathscr{R}_{11}, \mathscr{R}_{12}, \mathscr{R}_{21}, \mathscr{R}_{22} \in \mathbb{C}^{2,2}$, is biholomorphically equivalent to the set $\mathscr{A} \backslash \mathscr{B}$, where the algebraic sets $\mathscr{A}$ and $\mathscr{B}$ are specified below.

First, denote

$$
\mathscr{R}_{11}=A, \quad \mathscr{R}_{21}=B, \quad \mathscr{R}_{12}=C, \quad \mathscr{R}_{22}=D .
$$

Then Eqs. (6.10), (6.11) can be rewritten

$$
\begin{gathered}
\operatorname{tr} A=\operatorname{tr} B=\operatorname{tr} C=\operatorname{tr} D+\operatorname{det} A=0, \\
s_{1} \operatorname{tr} D-\operatorname{tr} A B=t_{1} \operatorname{tr} D-\operatorname{tr} A C=0, \\
s_{2} \operatorname{tr} D+\operatorname{det} B=t_{2} \operatorname{tr} D+\operatorname{det} C=s_{2} t_{2} \operatorname{tr} D+\operatorname{det} D=0, \\
s_{1} t_{1} \operatorname{tr} D-\operatorname{tr}(A D+B C)=0, \\
s_{1} t_{2} \operatorname{tr} D-\operatorname{tr} C D=s_{2} t_{1} \operatorname{tr} D-\operatorname{tr} B D=0 .
\end{gathered}
$$

The algebraic set $\mathscr{A}$ is the eight-dimensional irreducible component of the algebraic set (8.2) which is determined on the open set $\operatorname{tr} D \neq 0$ by the additional equations:

$$
s_{1} A C-A D-B C=s_{2} D B-B D=0 .
$$

In other words let $\mathscr{A}^{\prime}$ be the algebraic subset in the open set $\{\operatorname{tr} D \neq 0\} \subset \mathbb{C}^{20}$ which is determined by Eqs. (8.2), (8.3). Then the closure of $\mathscr{A}^{\prime}$ in $\mathbb{C}^{20}$ is exactly $\mathscr{A}$. The algebraic set $\mathscr{B}$ is contained in the hyperplane $\operatorname{tr} D=0$.

It remains to specify $\mathscr{B}$ and the intersection of $\mathscr{A}$ with this hyperplane. The Eq. (8.2) together with the condition $\operatorname{tr} D=0$ imply:

$$
X Y=0 \text { for arbitrary two matrices } X, Y \in\{A, B, C, D\} \text {. }
$$

This means that the matrices $A, B, C, D$ are multiples of the same nilpotent matrix. Let $\left\{E_{1}, E_{2}, E_{3}, E_{4}\right\}$ be a basis in $\overline{\mathfrak{g}} \bar{l}(2, \mathbb{C})$. For an element $E$ from this basis we denote

$$
a=\operatorname{tr} A E, \quad b=\operatorname{tr} B E, \quad c=\operatorname{tr} C E, \quad d=\operatorname{tr} D E .
$$

Then the intersection of $\mathscr{A}$ with the hyperplane $\operatorname{tr} D=0$ is determined by the equations

$$
\begin{gathered}
\left(c^{2}-a c t_{1}+a^{2} t_{2}\right) s_{1}-2 c d+(a d+b c) t_{1}-2 a b t_{2}=0 \\
\left(c^{2}-a c t_{1}+a^{2} t_{2}\right) s_{2}-d^{2}+b d t_{1}-b^{2} t_{2}=0, \\
\left(b^{2}-a b s_{1}+a^{2} s_{2}\right) t_{1}-2 b d+(a d+b c) s_{1}-2 a c s_{2}=0 \\
\left(b^{2}-a b s_{1}+a^{2} s_{2}\right) t_{2}-d^{2}+c d s_{1}-c^{2} s_{2}=0 .
\end{gathered}
$$

The algebraic set $\mathscr{B}$ is determined by the equations

$$
b^{2}-a b s_{1}+a^{2} s_{2}=c^{2}-a c t_{1}+a^{2} t_{2}=0 .
$$




\section{References}

1. Ward, R.: On self-dual gauge fields. Phys. Lett. 61 A, 81-82 (1977)

2. Yang, C.N.: Condition of self-duality for $S U(2)$ gauge fields on Euclidean four-dimensional space. Phys. Rev. Lett. 38, 1377-1379 (1977)

3. Belavin, A.A., Zakharov, V.G.: Yang-Mills equations as inverse scattering problem. Phys. Lett. 73 B, 53-57 (1978)

4. Atiyah, M., Hitchin, N., Singer, I.: Self-duality in four dimensional Riemannian geometry. Proc. R. Soc. Lond. A 362, 425-461 (1978)

5. Atiyah, M.F., Drinfeld, V., Hitchin, N.J., Manin, Yu.I.: Construction of instantons. Phys. Lett. 65 A, 185 (1978)

6. Corrigan, E.F., Fairlie, D.B., Yates, R.G., Goddard, P.: The construction of self-dual solutions to $S U(2)$ gauge theory. Commun. Math. Phys. 58, 223-240 (1978)

7. Pohlmeyer, K.: On the Lagrangian theory of anti-self-dual fields in four dimensional Euclidean space. Commun. Math. Phys. 72, 37-42 (1980)

8. Dolan, L.: A new symmetric group of real self-dual Yang-Mills theory. Phys. Lett. 113 B, 387-390 (1982)

9. Chau, L.L., Ge, M.L., Sinha, A., Wu, Y.S.: Hidden symmetry algebra for the self-dual YangMills equations. Phys. Lett. 121 B, 391 (1983)

10. Takasaki, K.: A new approach to the self-dual Yang-Mills equations. Commun. Math. Phys. 94, 35-59 (1984)

11. Donaldson, S.K.: Instantons and geometric invariant theory. Commun. Math. Phys. 93, 453-460 (1984)

12. Crane, L.: Action of the loop group on the self-dual Yang-Mills equation. Commun. Math. Phys. 110, 391-414 (1987)

13. Chau, L.L., Prasad, M.K., Sinha, A.: Some aspects of the linear system for self-dual YangMills fields. Phys. Rev. D 24, 1574 (1981)

14. Ward, R.S.: Ansätze for self-dual Yang-Mills fields. Commun. Math. Phys. 80, 563-574 (1981)

15. Uhlenbeck, K.: Removable singularities in Yang-Mills fields. Bull. Am. Math. Soc. 1, 579-581 (1979)

16. Okonek, C., Schneider, M., Spindler, H.: Vector bundles on complex projective spaces. Boston: Birkhäuser 1980

17. Hartshorne, R.: Algebraic geometry. Berlin, Heidelberg, New York: Springer 1977

18. Griffiths, P., Harris, J.: Principles of algebraic geometry. New York: John Wiley 1978

19. Hervé, M.: Several complex variables. Bombay: Oxford University Press 1963

20. Christ, N.H., Weinberg, E.J., Stanton, N.K.: General self-dual solutions. Phys. Rev. D 18, 2013-2025 (1978)

21. Hartshorne, R.: Stable vector bundles and instantons. Commun. Math. Phys. 59, 1-15 (1978)

22. van der Waerden, B.L.: Algebra I. Berlin, Heidelberg, New York: Springer 1971 